\title{
Generalized Daubechies Wavelet Families
}

\author{
Cédric Vonesch, Student Member, IEEE, Thierry Blu, Senior Member, IEEE, and Michael Unser, Fellow, IEEE
}

\begin{abstract}
We present a generalization of the orthonormal Daubechies wavelets and of their related biorthogonal flavors (Cohen-Daubechies-Feauveau, 9/7). Our fundamental constraint is that the scaling functions should reproduce a predefined set of exponential polynomials. This allows one to tune the corresponding wavelet transform to a specific class of signals, thereby ensuring good approximation and sparsity properties. The main difference with the classical construction of Daubechies $e t$ al. is that the multiresolution spaces are derived from scale-dependent generating functions. However, from an algorithmic standpoint, Mallat's Fast Wavelet Transform algorithm can still be applied; the only adaptation consists in using scale-dependent filter banks. Finite support ensures the same computational efficiency as in the classical case. We characterize the scaling and wavelet filters, construct them and show several examples of the associated functions. We prove that these functions are square-integrable and that they converge to their classical counterparts of the corresponding order.
\end{abstract}

Index Terms-Approximation order, biorthogonal, compact support, exponential polynomials, mutiresolution, nonstationary, orthonormal, reproduction, Strang-Fix, wavelet.

\section{INTRODUCTION}

\section{A. Scope of the Paper}

I $\mathrm{N}$ less than 20 years, wavelets have risen from a research curiosity to a standard signal processing tool in engineering and applied mathematics. While the mathematical foundations of wavelets and multiresolution analysis were laid down by Mallat and Meyer [1], [2], Daubechies had a lasting impact on the field with her construction of the first family of compactly supported, orthogonal wavelet bases of $L^{2}(\mathbb{R})$ [3]. Owing to their remarkable properties and ease of implementation, the Daubechies wavelets became popular right away and led to a multitude of successful signal processing applications, such as compression, denoising, classification, or fusion, especially during the wavelet rush that took place in the 1990s.

A good part of the success of wavelets is due to their fundamental vanishing moment property —or, equivalently, the ability of the scaling functions to reproduce polynomials. Indeed, the special way in which the basis functions interact with polynomials is the crucial ingredient that endows wavelets with their good approximation properties for signals in Sobolev and/or Besov spaces; in particular, it explains why piecewise smooth signals tend to have sparse wavelet expansions. This observation applies particularly well to the field of image processing,

Manuscript received July 21, 2006; revised January 24, 2007. This work was supported in part by the Swiss National Science Foundation under Grant 200020-101821. The associate editor coordinating the review of this mabuscript and approving it for publication was Dr. Gerald Schuller.

The authors are with the Biomedical Imaging Group, EPFL-STI-IOA-LIB, CH-1015 Lausanne, Switzerland (e-mail: cedric.vonesch@epfl.ch; thierry. blu@epfl.ch; michael.unser@epfl.ch).

Digital Object Identifier 10.1109/TSP.2007.896255 where wavelets have had (and are still having) a profound impact.

In this paper, we construct generalized wavelet bases that can be tuned to wider classes of signals, e.g., with multiple narrow bands or exponential trends. Still, we will retain the user-friendly properties of Daubechies wavelets; namely, compact support and othonormality (or biorthogonality if one also wants to include symmetry). Our starting point is the constraint that the scaling functions should reproduce a predefined set of exponential polynomials, that is, functions of the form $P(t) e^{\alpha t}$ where $P(t)$ is a polynomial and $\alpha$ is a complex parameter. For the aforementioned signal types, exponential polynomials ensure approximation properties that are comparable to those provided by standard polynomials for slowly varying signals.

\section{B. Illustration}

Our derivations have concrete implications for discrete signal processing, as illustrated in Fig. 1. We compare discrete orthonormal wavelet transforms of a signal made of two distinct frequency components. Classical 8-tap Daubechies filters were used in Fig. 1(a). We observe that a significant part of the energy is contained in the wavelet subbands, because the scaling filters are not suitable for the representation of pure sinusoids. For Fig. 1(b), the filters (of the same length) were adapted to the input signal, so that it gets transferred entirely in the scaling function subbands. From a practical standpoint, the only difference between (a) and (b) is that the latter uses scale-dependent filters. This shows that tuning our generalized wavelet bases to the class of signals to be decomposed can yield sparser representations than classical wavelets, at strictly the same computational cost.

\section{Relation With Existing Work}

We emphasize that our approach is quite different from the idea of wavelet packets [4]. In particular, the frequency behavior of wavelet packet basis functions cannot be easily controlled. While it is true that a wavelet packet tree can be designed to concentrate on a given frequency, one cannot guarantee that the resulting basis functions will be able to reproduce that precise frequency. It is even more difficult to make statements about the simultaneous reproduction of several distinct frequencies, or more generally, the reproduction of arbitrary exponentials.

The relations between classical wavelet theory and the properties of B-splines are well known [5]. Similarly, our generalized construction is closely related to exponential B-splines [6], [7]. The main mathematical result of this paper states that, for the scaling functions to reproduce exponential polynomials, their refinement filters must be divisible by scaling filters associated with exponential B-splines.

Because these filters are scale-dependent, we cannot use the framework of classical multiresolution spaces. Instead, our setting is based on nonstationary multiresolutions as introduced by de Boor, DeVore, and Ron [8]. In particular, this means that the 


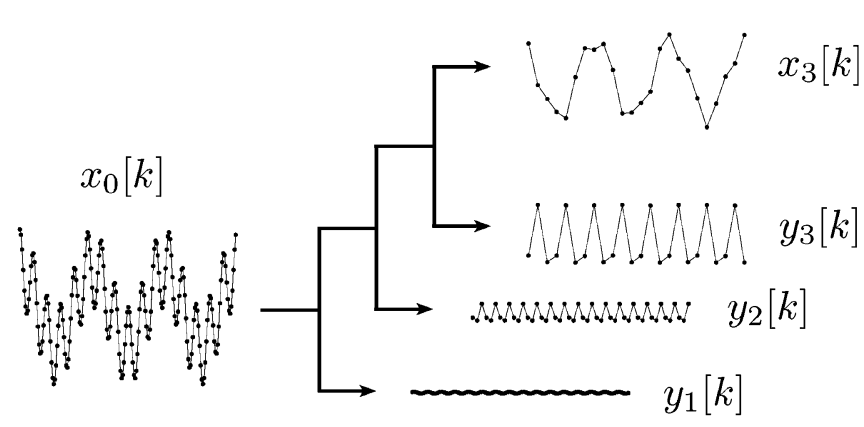

(a)

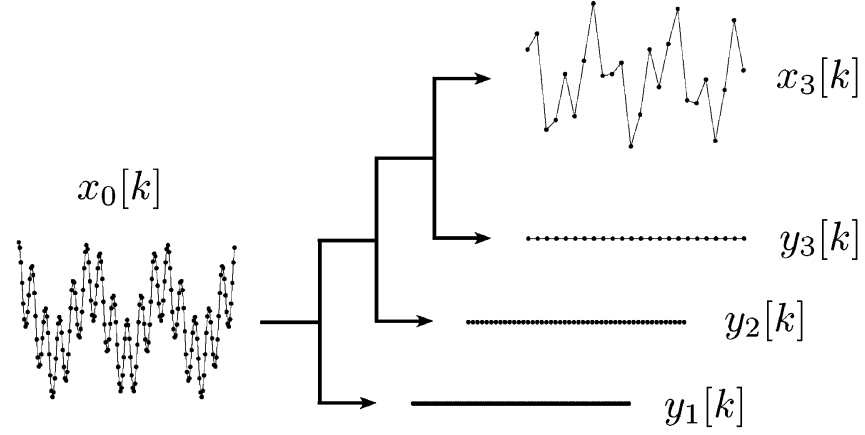

(b)

Fig. 1. Three-level discrete orthonormal wavelet transforms of a signal with two harmonic components $\left(x_{0}[k]=\cos (k \pi / 32)+\cos (k \pi / 6)\right)$. The wavelet coefficients are $y_{1}[k], y_{2}[k]$, and $y_{3}[k] ; x_{3}[k]$ contains the scaling function coefficients at the coarsest level. (a) Using 8-tap Daubechies filters (corresponding to $\vec{\alpha}=\left[\begin{array}{llll}0 & 0 & 0 & 0\end{array}\right]$ in Section IV-B). (b) Using scale-dependent 8-tap generalized Daubechies filters (corresponding to $\vec{\alpha}=[i \pi / 32-i \pi / 32 i \pi / 6-i \pi / 6]$ ).

scaling functions at different scales are not dilates of one another. This structure has already been used in previous work to construct dual bases of exponential B-splines [8], [9], with the important difference that the resulting functions are usually not compactly supported.

Another important connection can be established with nonstationary subdivision schemes [10]-[12], where the refinement filters are allowed to vary from one scale to the next. In particular, Dyn et al. [13] worked on generalized Deslauriers-Dubuc subdivision schemes [14]. The limit functions of these schemes can be seen as the autocorrelation of the generalized orthonormal Daubechies scaling functions that are constructed here.

Finally, from a filter-design perspective, this paper is concerned with conjugate quadrature filters that have arbitrarily placed zeros [15].

\section{Contents}

The paper is organized as follows. In Section II we give basic definitions and properties concerning nonstationary multiresolutions, including a quick review of wavelet theory in this generalized framework. In Section III we state the main theorem of this paper, which relates the reproduction of exponential polynomials to the roots of the scaling filters. Based on this result, we characterize the scaling filters of generalized Deslauriers-Dubuc interpolation functions in Section IV-A. Different factorizations of these filters are used in Sections IV-B and C to obtain the generalized Daubechies wavelets and scaling functions. In Section IV-D we prove the convergence of the infinite products defining these functions. In the Appendix, we provide a summary of the notations used in this paper, as well as proofs of its main results.

\section{NONSTATIONARY MULTIRESOlUtions}

\section{A. Definition}

The fundamental structure we are interested in is derived from the work of de Boor et al. [8].

Definition 1 (Nonstationary Multiresolution Spaces): Given an integer $j_{0}$ and a sequence of functions $\left(\varphi_{j}(t)\right)_{j \leq j_{0}}$ in $L^{2}(\mathbb{R})$, the spaces

$$
V_{j}=\left\{f(t)=\sum_{k \in \mathbb{Z}} c[k] \varphi_{j}\left(\frac{t-2^{j} k}{2^{j}}\right) \mid c \in \ell^{2}(\mathbb{Z})\right\}
$$

for $j \leq j_{0}$, define a nonstationary multiresolution if and only if

- for all $j \leq j_{0}, \varphi_{j}(t)$ generates a Riesz basis;

- for all $j \leq j_{0}-1, V_{j+1} \subset V_{j}$;

- $\bigcup_{j \leq j_{0}} V_{j}$ is dense in $L^{2}(\mathbb{R})$.

Note that in a classical (stationary) multiresolution [1], the functions $\left(\varphi_{j}(t)\right)_{j \leq j_{0}}$ would all be equal to a single function $\varphi(t)$ - the scaling function. Therefore, we shall call the functions $\left(\varphi_{j}(t)\right)_{j \leq j_{0}}$ scaling functions. In the sequel, to keep the notations simple, we will often omit the variable $t$ when referring to functions. Also, we will use a $\operatorname{dot}(\cdot)$ as a silent variable in $L^{2}(\mathbb{R})$-norms and scalar products.

\section{B. Basic Properties and Notations}

The Riesz basis constraint means that, at every scale $j \leq j_{0}$, there exist constants $0<B_{1 j} \leq B_{2 j}<+\infty$ such that, for all $c \in \ell^{2}(\mathbb{Z})$,

$$
\begin{aligned}
B_{1 j}\|c\|_{\ell^{2}(\mathbb{Z})} & \leq\left\|\sum_{k \in \mathbb{Z}} c[k] \varphi_{j}(\cdot-k)\right\|_{L^{2}(\mathbb{R})} \\
& \leq B_{2 j}\|c\|_{\ell^{2}(\mathbb{Z})}
\end{aligned}
$$

The values of the tightest pair $\left(B_{1 j}, B_{2 j}\right)$ are called lower and upper Riesz bounds, respectively. They are equal in the event of an orthogonal basis and their value is 1 if in addition the basis is normalized (i.e., orthonormal).

Throughout this paper, for a function $f \in L^{2}(\mathbb{R})$, we will use the notation $a_{f}[k]=\langle f, f(\cdot-k)\rangle=\int_{\mathbb{R}} f(t) f^{*}(t-k) \mathrm{d} t$ for its sampled autocorrelation. We shall also denote $C(z)=$ $\sum_{k \in \mathbb{Z}} c[k] z^{-k}$ the z-transform of a sequence $c$. We recall that the $\mathrm{z}$-transform of an autocorrelation sequence is always realvalued and positive over the unit circle (i.e., for $z=e^{i \omega}, \omega \in \mathbb{R}$ ).

With these notations, there is an alternative way to characterize the fact that a function $\varphi_{j}$ generates a Riesz basis: it is necessary and sufficient that there exist positive constants $B_{1 j}, B_{2 j}$ such that, for almost every $\omega \in \mathbb{R}$,

$$
0<B_{1 j}^{2} \leq A_{\varphi_{j}}\left(e^{i \omega}\right) \leq B_{2 j}^{2}<+\infty .
$$




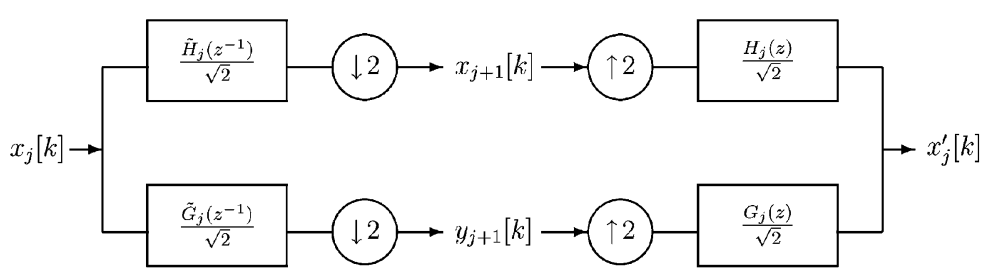

Fig. 2. Nonstationary perfect-reconstruction filter bank.

Moreover, the tightest-possible bounds $\left(B_{1 j}, B_{2 j}\right)$ are the same as those defined by (1).

The embedding of the spaces $V_{j}$ implies the existence of scaling filters $h_{j} \in \ell^{2}(\mathbb{Z})$ such that

$$
\varphi_{j+1}\left(\frac{t}{2^{j+1}}\right)=\sum_{k \in \mathbb{Z}} h_{j}[k] \varphi_{j}\left(\frac{t-2^{j} k}{2^{j}}\right) .
$$

As the notation suggests, the scaling filters are scale-dependent in general. One can also consider the frequency-domain version of the scaling relation (2), which is

$$
2 \hat{\varphi}_{j+1}(2 \omega)=H_{j}\left(e^{i \omega}\right) \hat{\varphi}_{j}(\omega) .
$$

Here, $\hat{f}(\omega)=\mathcal{F}\{f\}(\omega)=\int_{\mathbb{R}} f(t) e^{-i \omega t} \mathrm{~d} t$ denotes the Fourier transform of a function $f$.

\section{Wavelets, Biorthogonality, and Filter Banks}

In this paper, we will primarily be focused on the design of scaling functions that generate dual multiresolutions $\left(\tilde{V}_{j}\right)_{j \leq j_{0}}$ and $\left(V_{j}\right)_{j \leq j_{0}}$ (respectively the analysis and the synthesis spaces). This means that their basis functions are mutually biorthogonal $^{1}$

$$
\left\langle\varphi_{j}, \tilde{\varphi}_{j}(\cdot-k)\right\rangle=\delta[k]
$$

where $\delta[0]=1$ and $\delta[k]=0$ if $k \neq 0$.

Before going further, we shall briefly review the main ingredients of the corresponding (nonstationary) wavelet decompositions. Most of the material is analogous to the standard theory (see, e.g., [1], [16]), except for the important difference that the functions and the filters are now scale-dependent. To simplify the presentation, we will use the notations $\varphi_{j, k}(t)=$ $2^{-j / 2} \varphi_{j}\left(t / 2^{j}-k\right)$ and $\tilde{\varphi}_{j, k}(t)=2^{-j / 2} \tilde{\varphi}_{j}\left(t / 2^{j}-k\right)$ for all $j \leq j_{0}$ and all $k \in \mathbb{Z}$.

The following standard result gives a consequence of the biorthogonality for the scaling filters. It is given without proof [1].

Property 1: Assume that the scaling functions $\left(\tilde{\varphi}_{j}\right)_{j \leq j_{0}}$ and $\left(\varphi_{j}\right)_{j \leq j_{0}}$ generate multiresolutions as in Definition 1. Also assume that they are real-valued, compactly supported, and mutually biorthogonal for all $j \leq j_{0}$. Then, for all $j \leq j_{0}-1$, the scaling filters $\tilde{h}_{j}[k]$ and $h_{j}[k]$ have finite length and their $z$-transforms satisfy

$$
H_{j}(z) \tilde{H}_{j}\left(z^{-1}\right)+H_{j}(-z) \tilde{H}_{j}\left(-z^{-1}\right)=4 .
$$

From the scaling filters, one can construct the wavelet filters $G_{j}(z)=z^{2 m_{j}+1} \tilde{H}_{j}\left(-z^{-1}\right)$ and $\tilde{G}_{j}(z)=z^{2 m_{j}+1} H_{j}\left(-z^{-1}\right)$,

${ }^{1}$ We will always denote with a tilde " " the entities that are related to the dual spaces $\left(\tilde{V}_{j}\right)_{j \in \mathbb{Z}}$ (e.g., scaling functions, wavelets, filters, $\ldots$ ). where the integers $m_{j}$ can be chosen arbitrarily. Then the wavelets are given by

$$
\begin{aligned}
\psi_{j+1}(t) & =\sum_{k \in \mathbb{Z}} g_{j}[k] \varphi_{j}(2 t-k), \\
\tilde{\psi}_{j+1}(t) & =\sum_{k \in \mathbb{Z}} \tilde{g}_{j}[k] \tilde{\varphi}_{j}(2 t-k) .
\end{aligned}
$$

At each scale $j$, the dilated and shifted functions $\psi_{j, k}(t)=$ $2^{-j / 2} \psi_{j}\left(t / 2^{j}-k\right)$ and $\tilde{\psi}_{j, k}(t)=2^{-j / 2} \tilde{\psi}_{j}\left(t / 2^{j}-k\right)$ generate spaces $W_{j}$ and $\tilde{W}_{j}$, respectively; the previous definitions and the biorthogonality constraint imply that

$$
\left\{\begin{array}{l}
V_{j}=V_{j+1} \oplus W_{j+1} \text { and } W_{j+1} \perp \tilde{V}_{j+1} \\
\tilde{V}_{j}=\tilde{V}_{j+1} \oplus \tilde{W}_{j+1} \text { and } \tilde{W}_{j+1} \perp V_{j+1}
\end{array}\right.
$$

A $j_{0}$-scale wavelet decomposition of a function $f \in L^{2}(\mathbb{R})$ is given by

$$
f=\sum_{k \in \mathbb{Z}} x_{j_{0}}[k] \varphi_{j_{0}, k}+\sum_{j \leq j_{0}} \sum_{k \in \mathbb{Z}} y_{j}[k] \psi_{j, k}
$$

where $x_{j}[k]=\left\langle f, \tilde{\varphi}_{j, k}\right\rangle$ and $y_{j}[k]=\left\langle f, \tilde{\psi}_{j, k}\right\rangle$. This is the same formula as in the stationary theory, but one should keep in mind that the wavelets $\psi_{j, k}$ (respectively $\tilde{\psi}_{j, k}$ ) are no more dilates and translates of a single mother wavelet.

The filter bank in Fig. 2 implements the decomposition and reconstruction operations in terms of the discrete sequences $\left(x_{j}[k]\right)_{k \in \mathbb{Z}}$ and $\left(y_{j}[k]\right)_{k \in \mathbb{Z}}$ at the different scales $j$. The upper-left part of the filter bank computes $\left(x_{j+1}[k]\right)_{k \in \mathbb{Z}}$ from $\left(x_{j}[k]\right)_{k \in \mathbb{Z}}$, while the lower-left part yields the wavelet coefficients $\left(y_{j+1}[k]\right)_{k \in \mathbb{Z}}$. It is a perfect-reconstruction filter bank (i.e., $x_{j}^{\prime}[k]=x_{j}[k]$ for all $k \in \mathbb{Z}$ ) due to the biorthogonality relation (4) and the definition of the filters $\tilde{G}_{j}(z)$ and $G_{j}(z)$. The only difference with Mallat's fast wavelet transform [17] is that the filters depend on the scale parameter $j$.

\section{Exponential B-Spline Multiresolutions}

An example of nonstationary multiresolution spaces $\left(V_{j}\right)_{j \leq j_{0}}$ can be constructed from exponential B-splines [8]. We will summarize some of their basic properties here; a more extensive introduction can be found in [7].

Given a complex parameter-vector $\vec{\gamma} \in \mathbb{C}^{M}$, the corresponding exponential B-spline is defined in the frequency-domain as

$$
\hat{\beta}_{\vec{\gamma}}(\omega)=\prod_{m=1}^{M} \frac{1-e^{\gamma_{m}-i \omega}}{i \omega-\gamma_{m}} .
$$

Here, the variables $\gamma_{m}$ denote the components of $\vec{\gamma}$, for $m=1, \ldots, M$. In the time-domain, this yields a function $\beta_{\vec{\gamma}} \in L^{2}(\mathbb{R})$ whose support is $[0, M]$, as illustrated in Fig. 3 


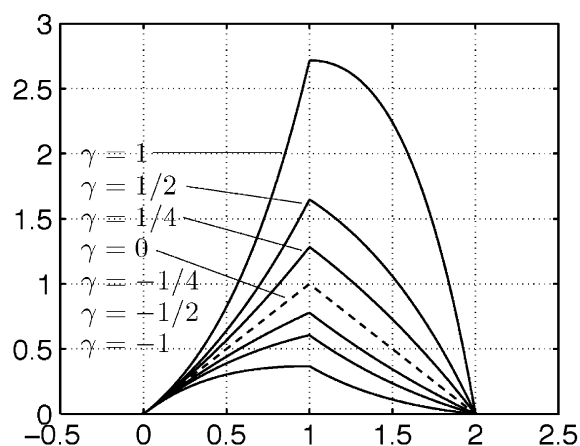

Fig. 3. Exponential B-splines $\beta_{\vec{\gamma}}(t)$ with parameter $\vec{\gamma}=[\gamma \gamma]$, where $\gamma$ is real. The classical second-order B-spline (the triangle function in dashed lines) is obtained for $\gamma=0$

for $M=2$. It is also worth noting that exponential B-splines tend to classical $M$ th-order B-splines as the parameter-vector $\vec{\gamma}$ tends to $\overrightarrow{0} \in \mathbb{C}^{M}$.

One can check that exponential B-splines satisfy the scaling relation

$$
2 \hat{\beta}_{2^{j+1} \vec{\gamma}}(2 \omega)=2^{1-M} R_{2^{j} \vec{\gamma}}\left(e^{i \omega}\right) \hat{\beta}_{2^{j} \vec{\gamma}}(\omega)
$$

where

$$
R_{\vec{\gamma}}(z)=\prod_{m=1}^{M}\left(1+e^{\gamma_{m}} z^{-1}\right) .
$$

In the aforementioned framework, this corresponds to $\varphi_{j}(t)=$ $\beta_{2^{j} \vec{\gamma}}(t)$ and $H_{j}(z)=2^{1-M} R_{2^{j} \vec{\gamma}}(z)$. We can thus consider the embedded shift-invariant spaces defined by

$$
V_{j}=\left\{f(t)=\sum_{k \in \mathbb{Z}} c[k] \beta_{2^{j}} \vec{\gamma}\left(\frac{t-2^{j} k}{2^{j}}\right) \mid c \in \ell^{2}(\mathbb{Z})\right\} .
$$

It is shown in [7] that, at a fixed scale $j$, the function $\beta_{2^{j}}(t)$ generates a Riesz-basis if and only if $\vec{\gamma}$ has no distinct purely imaginary components $\gamma$ and $\gamma^{\prime}$ such that $2^{j}\left(\gamma-\gamma^{\prime}\right)=i 2 \pi k$, for some $k \in \mathbb{Z}$. If this condition is satisfied at all scales $j$, then we can consider infinitely coarse spaces $V_{j}\left(j_{0}=+\infty\right)$. Otherwise we can only go up to some finite scale $j_{0}$, which is actually what is always done in practical applications of multiresolution analysis (only a finite number of decomposition levels is considered).

The density of the exponential B-spline spaces in $L^{2}(\mathbb{R})$ is proven in [9].

\section{E. Goals of the Present Paper}

Our aim is to design nonstationary multiresolution spaces that generalize the constructions of Daubechies [3], Cohen, Daubechies and Feauveau [18] and Antonini, Barlaud, Mathieu and Daubechies [19]. The first case corresponds to orthonormal scaling functions, while the latter two correspond to a biorthogonal setting. In all three cases, the scaling functions are compactly supported and real-valued; in addition, they have to be able to reproduce a predefined set of exponential polynomials.

We summarize these constraints on the scaling functions in the biorthogonal case, which is more general; the orthonormal case is obtained when enforcing $\tilde{\varphi}_{j}=\varphi_{j}$. At all scales $j$, we ask that

1) $\varphi_{j}$ and $\tilde{\varphi}_{j}$ are real-valued;

2) $\left\langle\varphi_{j}, \tilde{\varphi}_{j}(\cdot-k)\right\rangle=\delta[k]$;

3) $\varphi_{j}$ and $\tilde{\varphi}_{j}$ are compactly supported;

4) $\left(\varphi_{j, k}\right)_{k \in \mathbb{Z}}$ and $\left(\tilde{\varphi}_{j, k}\right)_{k \in \mathbb{Z}}$ each reproduce a given set of exponential polynomials (which does not depend on the scale j).

Our approach is constructive: we will assume that the above constraints are satisfied in order to derive necessary (and possibly sufficient) conditions for the scaling filters $\left(h_{j}[k]\right)_{j \leq j_{0}-1}$ and $\left(\tilde{h}_{j}[k]\right)_{j \leq j_{0}-1}$. We will then determine the shortest-possible filters that satisfy these conditions. Finally, we will derive the corresponding scaling functions in the frequency domain by iterating the scaling relation (3). For time-domain representations, we approximate the functions using numerical schemes comparable to the ones studied in [10], [12].

\section{REPRODUCTION OF EXPONENTIAL POLYNOMIALS}

\section{A. Preliminary Definitions}

We now introduce two key notions for the present formulation.

Definition 2 (Reproduction): A family of functions $\left(f_{k}\right)_{k \in \mathbb{Z}}$ reproduces a function $f$ if and only if there exists a sequence $(c[k])_{k \in \mathbb{Z}}$ such that the equality

$$
f(t)=\sum_{k \in \mathbb{Z}} c[k] f_{k}(t)
$$

holds almost everywhere.

Definition 3 (Fourier-Order): A compactly supported function $\Phi \in L^{2}(\mathbb{R})$ is said to be of order $L$ if and only if its Fourier transform satisfies

$$
\forall \ell=0, \ldots, L-1, \forall k \in \mathbb{Z} \backslash\{0\}, \hat{\Phi}^{(\ell)}(2 \pi k)=0
$$

or, equivalently,

$$
\forall k \in \mathbb{Z} \backslash\{0\}, \hat{\Phi}(\omega+2 \pi k)=O\left(\omega^{L}\right) .
$$

The present definition of order becomes equivalent to the classical Strang-Fix conditions [20] if one adds the requirement that $\hat{\Phi}(0) \neq 0$. This latter condition is automatically satisfied if $\Phi$ is of order $L \geq 1$ and generates a Riesz basis. However, one of the crucial aspects in the forthcoming mathematical results is that the function $\Phi$ under consideration does not necessarily generate a Riesz basis.

In what follows we will also denote by $\mathbb{C}[X]$ (respectively, $\mathbb{R}[X]$ ) the space of all polynomials of the variable $X$ with complex (respectively, real) coefficients. $\mathbb{C}_{L}[X]$ will stand for the polynomials of $\mathbb{C}[X]$ of degree less or equal to $L$.

\section{B. Relation With Exponential Vanishing Moments}

Before dealing with the connections between the above notions, we make a straightforward observation in the context of dual multiresolution spaces $\left(V_{j}\right)_{j \leq j_{0}},\left(\tilde{V}_{j}\right)_{j \leq j_{0}}$, generated by compactly supported scaling functions: imposing that the scaling functions must reproduce a given set of exponential polynomials implies exponential vanishing moments for the (dual) wavelets. 
Indeed, let us consider an exponential parameter $\gamma \in \mathbb{C}$ and a polynomial $P(X) \in \mathbb{C}[X]$. Assume that we can write

$$
P(t) e^{\gamma t}=\sum_{k \in \mathbb{Z}} c[k] \varphi_{j, k}(t)
$$

using some coefficient sequence $c . \varphi_{j}$ and $\tilde{\psi}_{j}$ are compactly supported, hence

$$
\int_{\mathbb{R}} \tilde{\psi}_{j, \ell}(t) P(t) e^{\gamma t} \mathrm{~d} t=\sum_{k=K_{1}}^{K_{2}} c[k]\left\langle\tilde{\psi}_{j, \ell}, \varphi_{j, k}\right\rangle=0
$$

for some integers $K_{1} \leq K_{2}$; the inner-products on the righthand side (RHS) are all zero because the functions $\tilde{\psi}_{j}$ and $\varphi_{j}$ (and all their integer shifts) are orthogonal.

\section{Generalized Strang-Fix Conditions}

Next we state and prove a generalized version of the Strang-Fix conditions for the reproduction of exponential polynomials.

Property 2 (Generalized Strang-Fix Conditions): Assume that $\varphi \in L^{2}(\mathbb{R})$ is compactly supported and define $\Phi(t)=$ $e^{-\gamma t} \varphi(t)$. If the family $(\varphi(t-k))_{k \in \mathbb{Z}}$ reproduces the exponential polynomials $P(t) e^{\gamma t}$, where $P(X) \in \mathbb{C}_{L-1}[X]$, then $\Phi$ is of Fourier-order $L$. The converse is true if $\hat{\Phi}(0) \neq 0$.

Proof:

- Direct part: For any polynomial $P(X) \in \mathbb{C}_{L-1}[X]$, there exist coefficients $(c[k])_{k \in \mathbb{Z}}$ such that

$$
\sum_{k \in \mathbb{Z}} c[k] \Phi(t+k)=P(t)
$$

almost everywhere. Since $\Phi$ is compactly supported, for all $t^{\prime}$ and almost all $t$ - and in particular for almost all $\left(t, t^{\prime}\right) \in$ $\mathbb{R}^{2}$-we can write

$$
\begin{aligned}
\sum_{n \in \mathbb{Z}}\left[\sum_{k \in \mathbb{Z}} c[k] \Phi(t-n+k)\right] \Phi\left(t^{\prime}+n\right) & \\
& =\sum_{n \in \mathbb{Z}}[P(t-n)] \Phi\left(t^{\prime}+n\right) .
\end{aligned}
$$

Introducing the new index $n^{\prime}=k-n$ yields

$$
\begin{aligned}
\sum_{n \in \mathbb{Z}} \sum_{n^{\prime} \in \mathbb{Z}} c\left[n+n^{\prime}\right] \Phi(t+ & \left.n^{\prime}\right) \Phi\left(t^{\prime}+n\right) \\
& =\sum_{n \in \mathbb{Z}} P(t-n) \Phi\left(t^{\prime}+n\right) .
\end{aligned}
$$

We observe that switching $t$ and $t^{\prime}$ does not change the left-hand side (LHS) expression. Therefore

$$
\sum_{n \in \mathbb{Z}} P\left(t^{\prime}-n\right) \Phi(t+n)=\sum_{n \in \mathbb{Z}} P(t-n) \Phi\left(t^{\prime}+n\right) .
$$

We denote by $S_{m}$ the LHS when $P(X)=X^{m}$. For $\ell \leq$ $L-1$, one can then compute

$$
\sum_{m=0}^{\ell}\left(\begin{array}{c}
\ell \\
m
\end{array}\right)\left(t+t^{\prime}\right)^{\ell-m}(-1)^{m} S_{m}
$$

in two different ways. After exchanging the $\sum_{n}$ and $\sum_{m}$ symbols and using the binomial identity, this yields

$$
\sum_{n \in \mathbb{Z}}(t+n)^{\ell} \Phi(t+n)=\sum_{n \in \mathbb{Z}}\left(t^{\prime}+n\right)^{\ell} \Phi\left(t^{\prime}+n\right) .
$$

Integrating this equation over $t^{\prime} \in[0,1]$ gives

$$
\sum_{n \in \mathbb{Z}}(t+n)^{\ell} \Phi(t+n)=\mu_{\ell}
$$

almost everywhere, where $\mu_{\ell}=\int_{-\infty}^{\infty} t^{\prime \ell} \Phi\left(t^{\prime}\right) \mathrm{d} t^{\prime}$ is a constant. Both sides are 1-periodic $L^{2}([0,1])$-functions. Equivalently, their Fourier-series coefficients satisfy

$$
\forall k \in \mathbb{Z}, i^{\ell} \hat{\Phi}^{(\ell)}(2 \pi k)=\mu_{\ell} \delta[k]
$$

for $\ell \leq L-1$. This demonstrates the Fourier-order property of Definition 3.

- Converse part: We use the same equivalence for the converse part of the proof. For $\ell \leq L-1$

$$
\begin{aligned}
& \sum_{n \in \mathbb{Z}} n^{\ell} \Phi(t+n) \\
& \quad=\sum_{n \in \mathbb{Z}}(t+n-t)^{\ell} \Phi(t+n) \\
& =\sum_{n \in \mathbb{Z}}\left[\sum_{m=0}^{\ell}\left(\begin{array}{c}
\ell \\
m
\end{array}\right)(t+n)^{\ell-m}(-t)^{m}\right] \Phi(t+n) \\
& =\mu_{0}(-t)^{\ell}+\sum_{m=0}^{\ell-1} \mu_{\ell-m}\left(\begin{array}{c}
\ell \\
m
\end{array}\right)(-t)^{m}
\end{aligned}
$$

The last expression is exactly a polynomial of degree $\ell$, since $\mu_{0}=\hat{\Phi}(0) \neq 0$. We conclude that the family $(\Phi(t-$ $k))_{k \in \mathbb{Z}}$ can reproduce a basis of $\mathbb{C}_{L-1}[t]$.

\section{Characterization of the Roots of the Scaling Filters}

We now arrive at a central result of this paper, which gives necessary and sufficient conditions on the scaling filters for the reproduction of exponential polynomials. At this point, we have to introduce two hypotheses on the scaling functions.

- H1 (Bounded asymptotic support): for $j \leq j_{0}$, the supports of the functions $\varphi_{j}(t)$ are included in some bounded interval $\left[t_{1}, t_{2}\right]$.

- $\mathbf{H 2}$ (Asymptotic Riesz condition): for $j \leq j_{0}$, the Riesz bounds $\left(B_{1 j}, B_{2 j}\right)$ of the functions $\varphi_{j}(t)$ are in some interval $\left[\mathcal{B}_{1}, \mathcal{B}_{2}\right]$ such that $0<\mathcal{B}_{1} \leq \mathcal{B}_{2}<+\infty$.

Theorem 1: Let $\left(\varphi_{j}(t)\right)_{j \leq j_{0}}$ be real-valued compactly supported functions in $L^{2}(\mathbb{R})$ that satisfy $\mathbf{H 1}$ and $\mathbf{H 2}$ and generate nonstationary multiresolution spaces as in Definition 1 . Also assume that, for all $j \leq j_{0}-1$, the filters $h_{j}[k]$ have finite length and that their z-transforms do not have pairs of opposite roots. Then the following statements are equivalent:

1) for all $j \leq j_{0}$, the functions $\left(\varphi_{j, k}\right)_{k \in \mathbb{Z}}$ reproduce the exponential polynomials $P(t) e^{\gamma t}$, where $\operatorname{deg} P(t) \leq L-1$;

2) for all $j \leq j_{0}-1$, the filter $H_{j}(z)$ has a zero of order $L$ at $z=-e^{2^{j} \gamma}$.

The proof is given in the Appendix. 


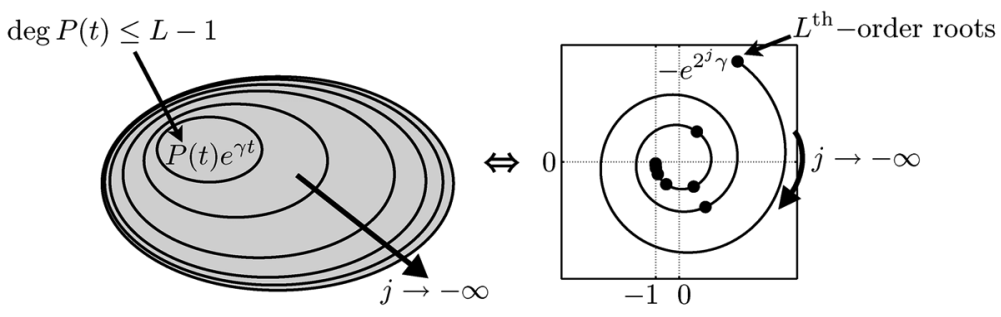

Fig. 4. Illustration of Theorem 1. For the exponential polynomials to lie in the linear span of the functions $\left(\varphi_{j, k}\right)_{k \in \mathbb{Z}}$ at all scales $j$, the scaling filters $H_{j}(z)$ must have roots located along a logarithmic spiral converging towards -1 in the complex plane.

Fig. 4 illustrates this theorem. In particular, in the stationary case (that is, when $\gamma=0$ ), one retrieves the classical result that polynomials of degree $L-1$ are reproduced if and only if the scaling filter has an $L$ th-order root at $z=-1$.

We can use this result to characterize scaling functions and filters that reproduce exponential polynomials with different parameters. To this end we let $\vec{\gamma}=\left[\begin{array}{lll}\gamma_{1} & \ldots & \gamma_{M}\end{array}\right]$ be a vector in $\mathbb{C}^{M}$ and we denote $L_{m}$ the multiplicity of element $\gamma_{m}$, for $m=1, \ldots, M$. We will define the exponential polynomials of parameter $\vec{\gamma}$ as the functions of the form

$$
P(t) e^{\gamma_{m} t}
$$

where $P(X)$ is a polynomial of degree less than $\left(L_{m}-1\right)$. Under the conditions of Theorem 1, for these exponential polynomials to be reproduced, it is necessary and sufficient that, for all $j \leq j_{0}-1, H_{j}(z)$ be divisible by $R_{2^{j} \vec{\gamma}}(z)$, as defined by (6). The shortest scaling functions that satisfy this condition are the exponential B-splines $\left(\beta_{2^{j} \vec{\gamma}}(t)\right)_{j \leq j_{0}}$, whose refinement filters are precisely $\left(R_{2^{j} \vec{\gamma}}(z)\right)_{j \leq j_{0}-1}$ [up to the multiplicative constant $2^{1-M}$, cf. (5)].

It is important to notice that the theorem assumes that, for all $j, H_{j}(z)$ has no roots of opposite sign. This condition arises naturally when imposing the biorthogonality constraint of Section II-C, (4), or the interpolation constraint of the next section (9). In terms of the vector $\vec{\gamma}$, it means that it cannot comprise elements $\gamma$ and $\gamma^{\prime}$ such that $2^{j}\left(\gamma-\gamma^{\prime}\right)=i(2 k+1) \pi$ for some $j \leq j_{0}-1$ and some $k \in \mathbb{Z}$. In what follows, we will refer to this constraint as $\mathbf{H 0}$.

\section{Construction of Generalized MulTiRESOLUTION BASES}

\section{A. Generalized Deslauriers-Dubuc Interpolation Functions}

As an application of the previous theorem, it is of interest to first consider the case where the scaling functions $\left(\varphi_{j}\right)_{j \leq j_{0}}$ are continuous and interpolating; i.e., for any $j \leq j_{0}$ and for any $k \in \mathbb{Z}$,

$$
\varphi_{j}(k)=\delta[k] .
$$

In this case, an exponential polynomial $P(t) e^{\gamma_{m} t}, \operatorname{deg} P(t) \leq$ $L_{m}-1$, can be easily written under the form

$$
P(t) e^{\gamma_{m} t}=\sum_{k \in \mathbb{Z}} \underbrace{P\left(2^{j} k\right) e^{2^{j} \gamma_{m} k}}_{c[k]} \varphi_{j}\left(t / 2^{j}-k\right)
$$

where the RHS coefficients $(c[k])_{k \in \mathbb{Z}}$ are simply the samples of the exponential polynomial at the locations $\left(2^{j} k\right)_{k \in \mathbb{Z}}$.
The interpolation property (7) imposes the following constraint on the scaling filters [16]:

$$
H_{j}(z)+H_{j}(-z)=2 .
$$

In addition, from Theorem $1, H_{j}(z)$ must be of the form $H_{j}(z)=R_{2^{j} \vec{\gamma}}(z) Q_{j}(z)$. We will thus look for the shortest-possible filters $Q_{j}(z)$ that satisfy

$$
R_{2^{j} \vec{\gamma}}(z) Q_{j}(z)+R_{2^{j} \vec{\gamma}}(-z) Q_{j}(-z)=2 .
$$

Equation (10) was studied by Dyn et al. in the context of nonstationary subdivision schemes [13]. In particular, they showed that, if $\vec{\gamma}$ satisfies condition H0, then there is a unique so-called minimal-rank solution $Q_{j}(z)$ at all scales $j \leq j_{0}-1$ (see also [15] for a more general analysis).

This result will be useful for the design of the orthonormal and biorthogonal structures in Sections IV-B and C. In order to provide some self-containedness while keeping the presentation simple, we will prove it in the case where $H_{j}(z)=R_{2^{j} \vec{\gamma}}(z) Q_{j}(z)$ is symmetric. The symmetry condition $H_{j}(z)=H_{j}\left(z^{-1}\right)$ allows us to choose $\vec{\gamma}$ such that, if 0 is an element of $\vec{\gamma}$, then it has even multiplicity. Moreover we are looking for real filters, therefore, we can choose $\vec{\gamma}$ such that, if $\gamma$ is an element of $\vec{\gamma}$, then so are $\gamma^{*},-\gamma$ and $-\gamma^{*}$, with the same multiplicity. In particular, this implies that $M$ is even. We can then perform the standard change of variable $Z=\left(z+z^{-1}\right) / 2$ and construct polynomials $C_{j}(Z), D_{j}(Z) \in \mathbb{R}[Z]$ such that

$$
\left\{\begin{array}{l}
z^{M / 2} R_{2^{j}}(z)=C_{j}\left(\frac{z+z^{-1}}{2}\right) ; \\
z^{-M / 2} Q_{j}(z)=D_{j}\left(\frac{z+z^{-1}}{2}\right) .
\end{array}\right.
$$

We are now ready to apply the following classical result.

Theorem 2 (Bézout): Given $C(Z) \in \mathbb{R}[Z]$, there exists a polynomial $D(Z) \in \mathbb{R}[Z]$ such that

$$
C(Z) D(Z)+C(-Z) D(-Z)=2
$$

if and only if $C(Z)$ has neither zero as a root, nor a pair of opposite roots. In this case there exists a unique polynomial $D_{0}(Z) \in$ $\mathbb{R}[Z]$ satisfying (12) and such that $\operatorname{deg} D_{0}(Z) \leq \operatorname{deg} C(Z)-1$. The set of all polynomials $D(Z) \in \mathbb{R}[Z]$ that satisfy (12) is

$$
\left\{D_{0}(Z)+Z \lambda\left(Z^{2}\right) C(-Z), \lambda(Z) \in \mathbb{R}[Z]\right\} .
$$

There are several possible ways to obtain $D_{0}(Z)$ in practice, e.g., via the resolution of a linear system (matrix inversion) or the extended Euclid algorithm. Another approach consists in rewriting (12) as in Fig. 5, by dividing it by $C(Z) C(-Z)$. $D_{0}(Z)$ can then be computed from a simple fraction decomposition of $2 /(C(Z) C(-Z))$, followed by a separation of the 


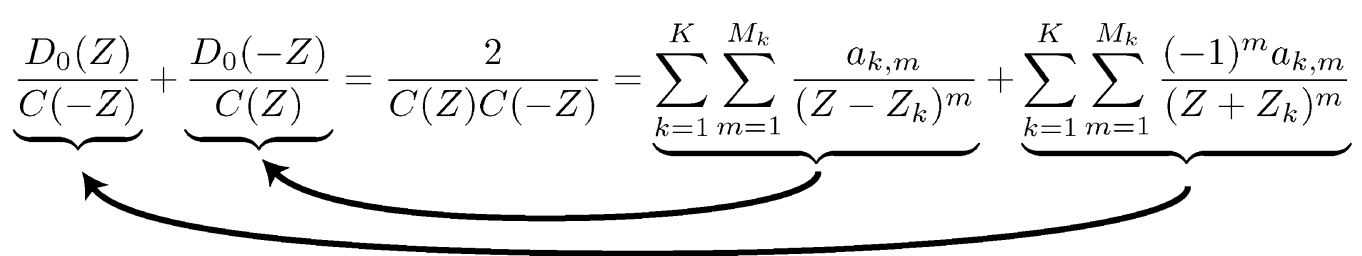

Fig. 5. How to obtain $D_{0}(Z)$, the lowest degree Bézout solution to (12).

simple fractions in two groups: one whose poles are the roots of $C(Z)$ and the other whose poles are the roots of $C(-Z)$. Because $C(Z)$ and $C(-Z)$ cannot have the same roots, $D_{0}(Z)$ is obtained by multiplying the second group by $C(-Z)$. Clearly, $\operatorname{deg} D_{0}(Z) \leq \operatorname{deg} C(Z)-1$ and we know that such a solution of (12) is unique. The method is summarized in Fig. 5, where the roots of $C(Z)$ are denoted $\left(Z_{k}\right)_{1 \leq k \leq K}$, with respective multiplicities $\left(M_{k}\right)_{1 \leq k \leq K}$. The symmetry of the decomposition is reflected by the simple fraction coefficients $a_{k, m}$ and $(-1)^{m} a_{k, m}$.

At each scale $j$, we will denote $D_{0 j}(Z)$ the lowest-degree Bézout solution corresponding to $C(Z)=C_{j}(Z)$ in (12); this is the polynomial $D_{j}(Z)$ we use to derive $Q_{j}(z)$ through (11). Fig. 6(a) shows $\varphi_{0}(t)$, the resulting scaling function at

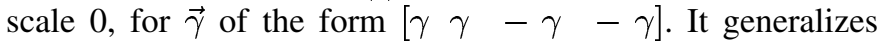
the Deslauriers-Dubuc interpolation function [14], [21] of the corresponding order $(M=4)$, which is obtained when $\gamma=0$. While the Deslauriers-Dubuc scaling function can reproduce polynomials up to degree 3 , our scaling function reproduces $P(t) e^{ \pm \gamma t}$ for $\operatorname{deg} P(t) \leq 1$ [Fig. 6(b)].

\section{B. Generalized Daubechies Wavelets}

We now return to our original problem as stated in Section II-E. Here, we denote by $\vec{\alpha} \in \mathbb{C}^{N}$ the ( $N$-dimensional) parameter-vector of the exponential polynomials to be reproduced. Since we are looking for real-valued filters, if $\alpha$ is a component of $\vec{\alpha}$, then so must $\alpha^{*}$, with the same multiplicity.

From Property 1 and Theorem 1, we get to the following design problem in terms of the scaling filters: we have to find the shortest-possible filters $Q_{j}(z)$ such that

$$
\left\{\begin{array}{l}
H_{j}(z) H_{j}\left(z^{-1}\right)+H_{j}(-z) H_{j}\left(-z^{-1}\right)=4 \\
H_{j}(z)=R_{2^{j} \vec{\alpha}}(z) Q_{j}(z)
\end{array}\right.
$$

This problem can be related to the one presented in the previous section by introducing the vector $\vec{\gamma}=(\vec{\alpha}:-\vec{\alpha})$, which has length $M=2 N$ (here ":" denotes concatenation). Indeed, at a fixed scale $j$, solving (14) is equivalent to the following:

1) solving for $D_{j}(Z)$ the Bézout equation $C_{j}(Z) D_{j}(Z)+$ $C_{j}(-Z) D_{j}(-Z)=2$, where

$$
C_{j}\left(\frac{z+z^{-1}}{2}\right)=z^{M / 2} R_{2^{j} \vec{\gamma}}(z)=c_{j} R_{2^{j} \vec{\alpha}}(z) R_{2^{j} \vec{\alpha}}\left(z^{-1}\right)
$$

is a symmetric Laurent polynomial with real coefficients and $c_{j}=\prod_{n=1}^{N} e^{-2^{j} \alpha_{n}}$ is a positive constant;

2) performing the spectral factorization

$$
2 c_{j} D_{j}\left(\frac{z+z^{-1}}{2}\right)=Q_{j}(z) Q_{j}\left(z^{-1}\right) .
$$

Note that, for the first step to be solvable at all scales, $\vec{\gamma}$ must satisfy condition $\mathbf{H O}$ (which is another way to state that $C_{j}(Z)$

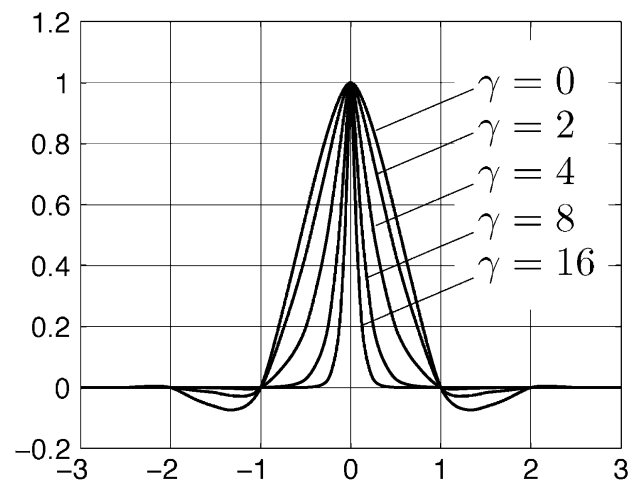

(a)

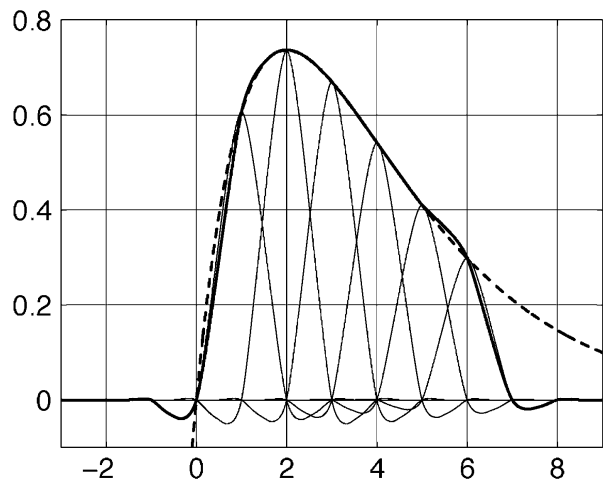

(b)

Fig. 6. (a) Interpolation functions at scale $j=0$ for $\vec{\gamma}=[\gamma \gamma-\gamma-\gamma]$. (b) Reproduction of the exponential polynomial $t e^{-t / 2}$ (dashed line) for $\gamma=$ $1 / 2$; the thick continuous line represents the sum in (8) truncated to $k=0 \ldots 6$, while its individual terms are shown as thin lines.

and $C_{j}(-Z)$ do not have common roots). The following result [22] states under which conditions the second step is possible.

Lemma 1 (Fejér-Riesz): Given a polynomial $S(Z) \in \mathbb{R}[Z]$, there exists a Laurent polynomial $Q(z)$ with real coefficients such that

$$
S\left(\frac{z+z^{-1}}{2}\right)=Q(z) Q\left(z^{-1}\right)
$$

if and only if $S(Z) \geq 0$ for $Z \in[-1,1]$.

Here $S(Z)=2 c_{j} D_{j}(Z)$ where $c_{j}$ is a positive constant. It is known that in the stationary case $\left(\vec{\alpha}=\overrightarrow{0} \in \mathbb{C}^{N}\right)$, the lowest degree solution of (12) is a polynomial that is positive over $[-\sqrt{2}, \sqrt{2}]$ (see e.g., [23] for a more detailed analysis of the roots of this type of polynomials). However, in our case, depending on the choice of the parameter-vector $\vec{\alpha}$, it may happen that the shortest Bézout solution $D_{0 j}(Z)$ is not positive over $[-1,1]$. We then have to look for higher-degree solutions in the set given by (13). We can show that, for a degree large enough, 


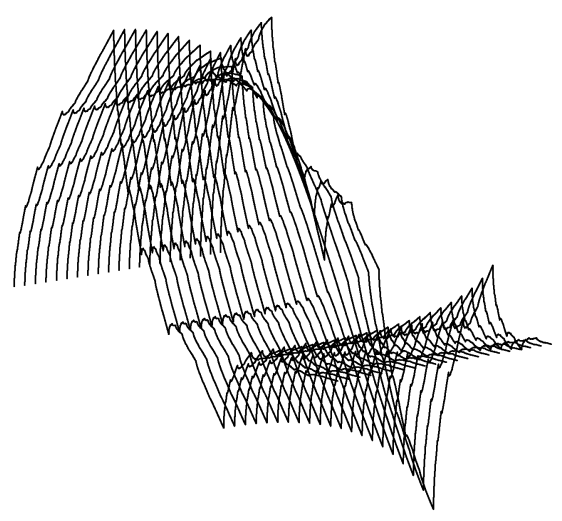

(a)

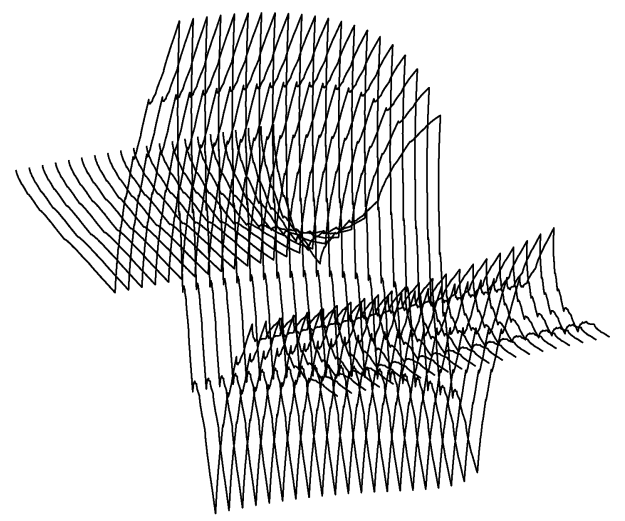

(b)

Fig. 7. Orthonormal scaling functions (a) and wavelets (b) at scale $j=0$, corresponding to $\vec{\alpha}=\left[i \omega_{0}-i \omega_{0}\right]$, for $\omega_{0}=0, \pi / 32,2 \pi / 32, \ldots, 20 \pi / 32$. The classical Daubechies functions are the leftmost ones and are obtained for $\omega_{0}=0$.

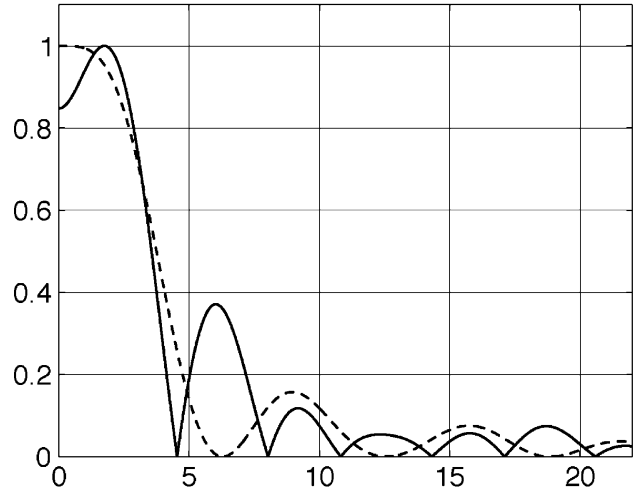

(a)

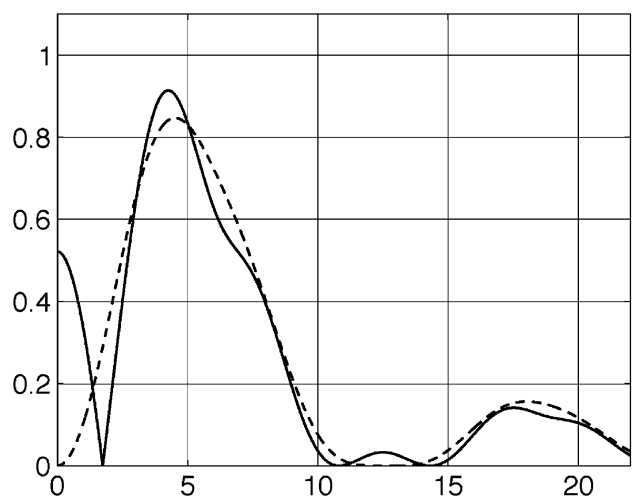

(b)

Fig. 8. Moduli of the Fourier transforms of the orthonormal scaling functions (a) and wavelets (b) at scale $j=0$, corresponding to $\vec{\alpha}=\left[\begin{array}{ll}i \omega_{0} & -i \omega_{0}\end{array}\right]$. The classical Daubechies functions are obtained for $\omega_{0}=0$ and are shown as dashed lines; their generalized counterparts are shown as continuous lines, for $\omega_{0}=5 \pi / 9$.

there always exists a positive solution. This allows us to generalize Daubechies's idea to look for a polynomial $D_{j}(Z)$ with lowest-possible degree (which might not be unique, though).

Property 3: If $C_{j}(Z) \in \mathbb{R}[Z]$ does neither have zero as a root, nor a pair of opposite roots, then there exists a polynomial $D_{j}(Z) \in \mathbb{R}[Z]$ such that

$$
\left\{\begin{array}{l}
C_{j}(Z) D_{j}(Z)+C_{j}(-Z) D_{j}(-Z)=2 ; \\
D_{j}(Z) \geq 0 \text { for } Z \in[-1,1] .
\end{array}\right.
$$

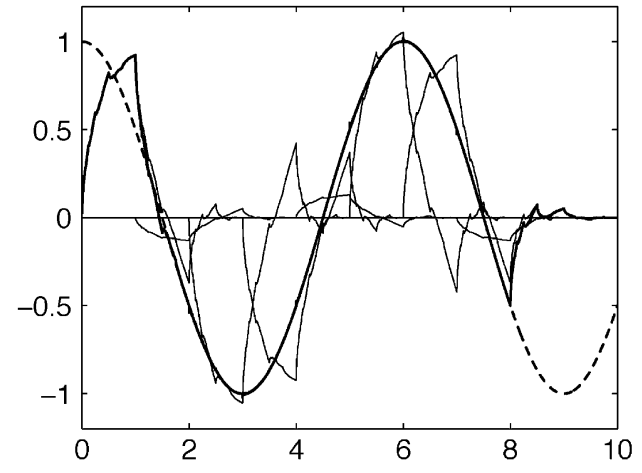

Fig. 9. Reproduction of $\cos (\pi t / 3)$ by the generalized orthonormal scaling function corresponding to $\vec{\alpha}=[i \pi / 3-i \pi / 3]$. Only eight terms in the sum are shown.

The proof, which is given in the Appendix, is simple and constructive: it uses entities related to exponential B-splines. Note that in the spectral factorization step (15), the roots of $Q_{j}(z)$ are always chosen inside the unit circle.

Fig. 7 shows plots of the resulting scaling functions and wavelets (with $\vec{\alpha}$ of the form $\left[i \omega_{0}-i \omega_{0}\right]$, where $\omega_{0}$ is a real frequency parameter). It also compares them with their classical counterparts (which are obtained for $\omega_{0}=0$ ). It is seen that the scaling functions and wavelets tend toward the standard second-order Daubechies functions as the vector $\vec{\alpha}$ tends to zero. Section IV-D will provide a more detailed proof of convergence. In the frequency-domain (Fig. 8), the second-order zeros at $\omega=2 \pi k$ for $k \in \mathbb{Z}^{*}$ of the standard Daubechies scaling functions are replaced by pairs of simple zeros at $\omega= \pm \omega_{0}+2 \pi k$. This is to satisfy the generalized Strang-Fix conditions, which guarantee that $e^{ \pm i \omega_{0} t}$ can be reproduced, and thus $\cos \left(\omega_{0} t\right)$ as well (see Fig. 9).

\section{Biorthogonal Spectral Factorization Wavelets}

In the biorthogonal case, the scaling filters must satisfy (4) and be of the form

$$
\left\{\begin{array}{l}
H_{j}(z)=R_{2^{j}}(z) Q_{j}(z) \\
\tilde{H}_{j}(z)=R_{2^{j} \overrightarrow{\tilde{\alpha}}}(z) \tilde{Q}_{j}(z) .
\end{array}\right.
$$


Here, the exponential polynomials to be reproduced are defined by the complex vectors $\vec{\alpha}$ and $\overrightarrow{\tilde{\alpha}}$, of length $N$ and $\tilde{N}$, respectively. A priori, the parameters for the analysis side and for the synthesis side can be freely chosen, provided the concatenated vector $\vec{\gamma}=(\vec{\alpha}:-\overrightarrow{\tilde{\alpha}})$ fulfills condition H0. Otherwise, the LHS of (4) would have a root in the complex plane, rendering the biorthogonality constraint impossible to satisfy. Also, to obtain real filters, one must make sure that, if $\alpha$ is an element of $\vec{\alpha}$ (respectively, $\overrightarrow{\widetilde{\alpha}}$ ), then the same must be true for $\alpha^{*}$, with the same multiplicity.

Furthermore, to keep the presentation simple, we will consider the case ${ }^{2}$ where $\vec{\gamma}$ satisfies the symmetry conditions of Section IV-A. That is, if 0 is an element of $\vec{\gamma}$, then it must have even multiplicity, and, if $\gamma$ is an element of $\vec{\gamma}$, then so are $\gamma^{*},-\gamma$ and $-\gamma^{*}$, with the same multiplicity. Similarly to what was done in the previous section, we can then determine $Q_{j}(z)$ and $\tilde{Q}_{j}(z)$ in two steps:

1) solving for $D_{0 j}(Z)$ the Bézout equation $C_{j}(Z) D_{0 j}(Z)+$ $C_{j}(-Z) D_{0 j}(-Z)=2$, where

$$
\begin{aligned}
C_{j}\left(\frac{z+z^{-1}}{2}\right) & =z^{M / 2} R_{2^{j} \vec{\gamma}}(z) \\
& =\tilde{c}_{j} z^{(N-\tilde{N}) / 2} R_{2^{j} \vec{\alpha}}(z) R_{2^{j} \vec{\alpha}}\left(z^{-1}\right)
\end{aligned}
$$

with $\tilde{c}_{j}=\prod_{n=1}^{\tilde{N}} e^{-2^{j} \tilde{\alpha}_{n}}$;

2) performing the spectral factorization

$$
2 z^{(N-\tilde{N}) / 2} \tilde{c}_{j} D_{0 j}\left(\frac{z+z^{-1}}{2}\right)=Q_{j}(z) \tilde{Q}_{j}\left(z^{-1}\right) .
$$

We point out that $Q_{j}(z) \neq \tilde{Q}_{j}(z)$, which relaxes the positivity constraint on $D_{j}(Z)$, and thus we can always use the lowestdegree Bézout solution $D_{0 j}(Z)$. However, as in the classical theory, not all potential factorizations of the type (16) lead to regular functions.

1) Generalized Cohen-Daubechies-Feauveau Wavelets: Cohen, Daubechies and Feauveau [18] specified biorthogonal wavelet bases derived from compactly supported duals of $\mathrm{B}$-spline functions. We can generalize this by constructing compactly supported duals of exponential B-splines. Accordingly, we constrain the filters $H_{j}(z)$ to be exponential B-spline scaling filters (cf. Section II-D): $H_{j}(z)=2^{1-N} R_{2^{j} \vec{\alpha}}(z)$. This means that $Q_{j}(z)=2^{1-N}$ and

$$
\tilde{Q}_{j}(z)=2^{N} z^{(\tilde{N}-N) / 2} \tilde{c}_{j} D_{0 j}\left(\frac{z+z^{-1}}{2}\right) .
$$

The resulting scaling functions $\varphi_{j}$ are exponential B-splines of parameter $2^{j} \vec{\alpha}$, that admit $\tilde{\varphi}_{j}$ as their compactly supported duals. The sum of their support lengths is minimal given the sets of exponential polynomials that they have to reproduce (which are specified by the parameters $\vec{\alpha}$ and $\overrightarrow{\tilde{\alpha}}$ ). Fig. 10 shows a small sample of the wide variety of scaling functions and wavelets that can be obtained (on the RHS) and allows for a comparison with their classical counterparts (on the LHS). Notice that, contrary to classical B-splines, exponential B-splines (and their duals) are not necessarily symmetric. But the user always has the option to make them symmetric by adequately choosing the parameters $\vec{\alpha}$ and $\overrightarrow{\tilde{\alpha}}$, as illustrated in Fig. 10(f). In the RHS examples, one can

${ }^{2}$ In the general case, one can derive $Q_{j}(z)$ and $\tilde{Q}_{j}(z)$ from the work of Dyn et al. [13].
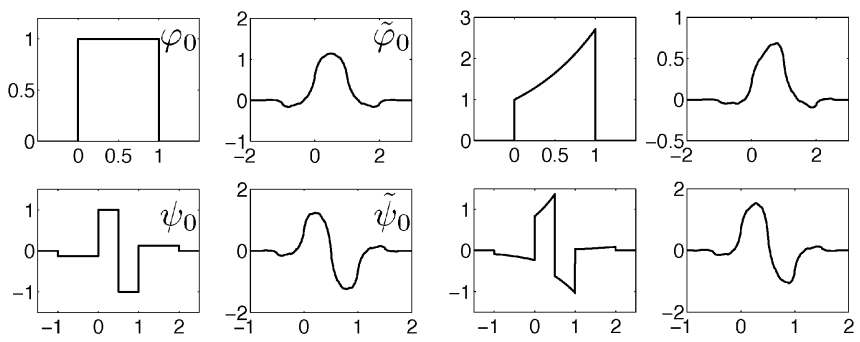

(a)

(b)
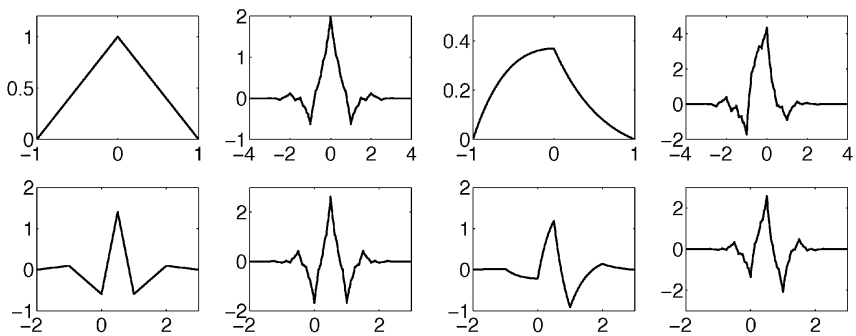

(c)
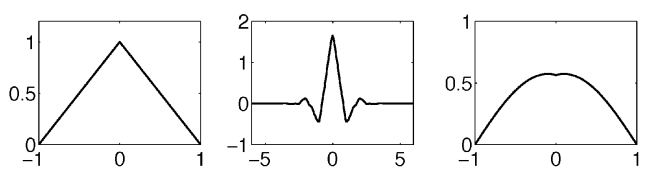

(d)
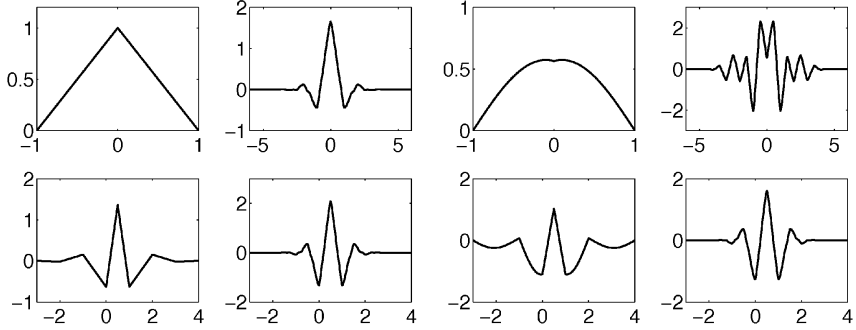

(e)

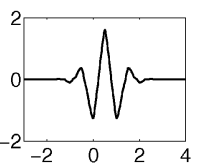

(f)

Fig. 10. Examples of Cohen-Daubechies-Feauveau functions (left) and of their generalized counterparts (right), for various combinations of $\vec{\alpha}$ and $\overrightarrow{\tilde{\alpha}}$. Each group shows (from left to right and top to bottom) $\varphi_{0}, \tilde{\varphi}_{0}, \psi_{0}$ and $\tilde{\psi}_{0}$. (a) $\vec{\alpha}=$ $[0] ; \overrightarrow{\tilde{\alpha}}=\left[\begin{array}{lll}0 & 0 & 0\end{array}\right]$. (b) $\vec{\alpha}=[1] ; \overrightarrow{\tilde{\alpha}}=\left[\begin{array}{lll}-1 & 1 & 1\end{array}\right]$. (c) $\vec{\alpha}=\left[\begin{array}{ll}0 & 0\end{array}\right] ; \overrightarrow{\tilde{\alpha}}=\left[\begin{array}{llll}0 & 0 & 0 & 0\end{array}\right]$. (d) $\vec{\alpha}=\left[\begin{array}{ll}-1 & -1\end{array}\right] ; \overrightarrow{\tilde{\alpha}}=\left[\begin{array}{llll}-1 & -1 & -1 & 1\end{array}\right]$. (e) $\vec{\alpha}=\left[\begin{array}{ll}0 & 0\end{array}\right] ; \overrightarrow{\tilde{\alpha}}=\left[\begin{array}{llllll}0 & 0 & 0 & 0 & 0 & 0\end{array}\right]$.

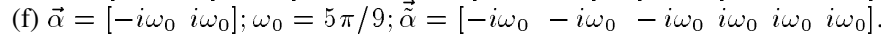

observe that the exponential trends (b), (d) and the oscillatory modes (f) influence the general shape of the functions.

This construction should be compared to the one proposed by Khalidov and Unser in [9]. Here, the authors also obtain duals of exponential B-splines, with the important difference that the former lie in the linear span of the latter. For this reason, the resulting functions are infinitely supported as soon as $N \geq 2$.

2) Generalized 9/7 Wavelets: The orthonormal setting described in Section IV-B does not permit the construction of symmetric basis functions (except for the Haar functions). As illustrated above, this limitation does not exist in the biorthogonal (nonorthonormal) setting. However, we can construct bases that are both symmetric and "closer" to orthogonality than the ones described in Section IV-C-1. As an example, we derive generalized biorthogonal 9/7 wavelets [19]. These are symmetric and their corresponding pairs of dual scaling functions have less disparate support lengths. These features are especially desirable in image processing applications [24].

The acronym " $9 / 7$ " refers to the lengths of the dual scaling filters $\tilde{h}_{j}, h_{j}$. In our notation they are obtained from vectors $\overrightarrow{\tilde{\alpha}}, \vec{\alpha}$ of dimension 4 each, together with a nontrivial factorization of the polynomial $D_{0 j}(Z)$ in (16). The factorization is performed with respect to the $Z$ variable so as to ensure that $Q_{j}(z)$ and $\tilde{Q}_{j}\left(z^{-1}\right)$ are symmetric. 

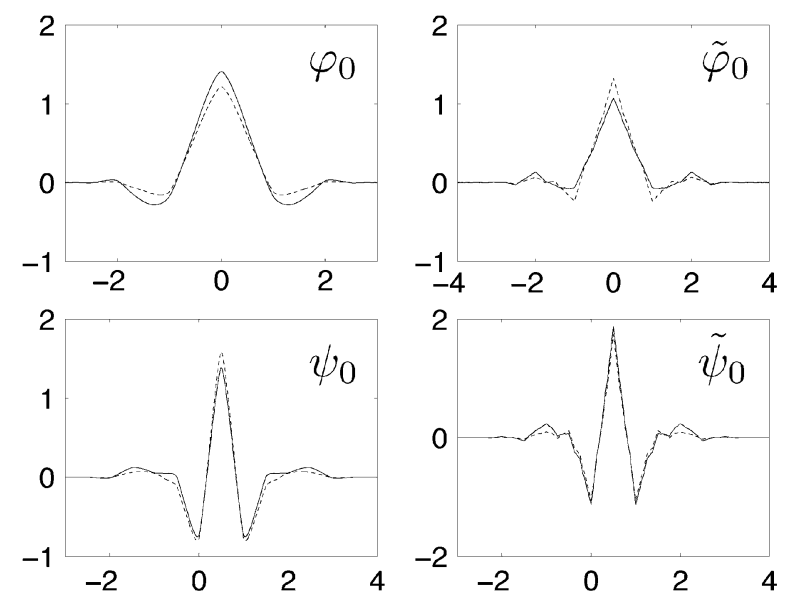

Fig. 11. Biorthogonal scaling functions and wavelets at scale 0 , corresponding to a generalization of the $9 / 7$ filters (see text). From left to right and top to bottom: $\varphi_{0}, \tilde{\varphi}_{0}, \psi_{0}$ and $\tilde{\psi}_{0}$. The functions obtained from the classical $9 / 7$ filters are represented using dashed lines.

For the sake of illustration, we take $\overrightarrow{\tilde{\alpha}}=\vec{\alpha}=\left[i \omega_{0} \quad i \omega_{0}\right.$ $\left.i \omega_{0}-i \omega_{0}\right]$. With that choice, there is a simple relation between the stationary case (once again obtained for $\omega_{0}=0$ ) and the case of an arbitrary parameter $\omega_{0}$. Namely

$$
C_{j}(Z)=\cos \left(2^{j} \omega_{0}\right)^{4} C\left(\frac{Z}{\cos \left(2^{j} \omega_{0}\right)}\right)
$$

where $C(Z)$ stands for the polynomial $C_{j}(Z)$ when $j \rightarrow-\infty$. Equivalently, this corresponds to the stationary case $\omega_{0}=0$. It follows that:

$$
D_{0 j}(Z)=\frac{1}{\cos \left(2^{j} \omega_{0}\right)^{4}} D_{0}\left(\frac{Z}{\cos \left(2^{j} \omega_{0}\right)}\right) .
$$

Here (and for the remainder of this paper), the notation $D_{0}(Z)$ refers to the lowest-degree Bézout solution in the stationary case. It is known that it is of degree 3 and has a pair of complex-conjugate roots. Thus the same holds for $D_{0 j}(Z)$ and there is only one nontrivial factorization leading to symmetric and real-valued filters $h_{j}$ and $\tilde{h}_{j}$. Note that we always normalize $Q_{j}(z)$ and $\tilde{Q}_{j}(z)$ such that $H_{j}(1)=\tilde{H}_{j}(1)$.

In Fig. 11, the functions obtained for $\omega_{0}=2 \pi / 5$ are shown and compared to the reference functions $\left(\omega_{0}=0\right)$. Fig. 12 compares the ratios of the Riesz bounds of generalized $9 / 7$ scaling functions and the corresponding generalized Cohen-Daubechies-Feauveau scaling functions, obtained when using the same parameters $\vec{\alpha}, \overrightarrow{\tilde{\alpha}}$ as above, for different values of $\omega_{0}$. It is seen that the ratios of the 9/7 scaling functions are always closer to 1 , indicating that they are closer to orthogonality than exponential B-spline scaling functions. It is also seen that in both cases the ratios are relatively stable up to $\omega_{0}=\pi / 4$.

\section{Convergence}

In this last part, we prove that the scaling filters derived before specify well-defined scaling functions in the continuous-time domain.

In view of the scaling relation (3), it is natural to define the scaling functions in the Fourier-domain as

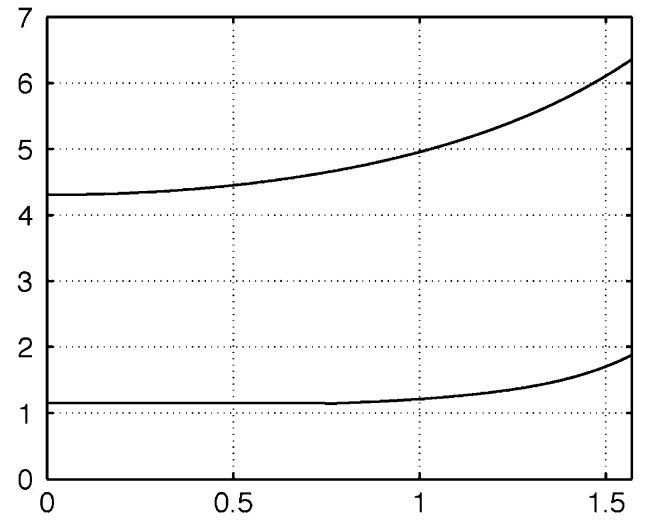

Fig. 12. Ratios of the Riesz bounds $B_{20} / B_{10}$ for Cohen-DaubechiesFeauveau (top) and $9 / 7$ (bottom) synthesis scaling functions at scale 0 , as a function of $\omega_{0}\left(\overrightarrow{\tilde{\alpha}}=\vec{\alpha}=\left[\begin{array}{llll}i \omega_{0} & i \omega_{0} & -i \omega_{0} & -i \omega_{0}\end{array}\right]\right)$.

$$
\hat{\varphi}_{j}(\omega)=\prod_{\ell=1}^{+\infty} \frac{1}{2} H_{j-\ell}\left(e^{i \omega / 2^{\ell}}\right) .
$$

A similar definition is used for the dual functions $\hat{\tilde{\varphi}}_{j}(\omega)$, and the results below also apply to them.

In what follows, we will drop the index " $j$ " when referring to entities that correspond to the stationary case (that is, to $\vec{\gamma}=\overrightarrow{0} \in$ $\mathbb{C}^{M}$, respectively, $\vec{\alpha}=\overrightarrow{0} \in \mathbb{C}^{N}$, respectively, $\left.\overrightarrow{\tilde{\alpha}}=\overrightarrow{0} \in \mathbb{C}^{\tilde{N}}\right)$. For example, in the context of Section IV-B, $\hat{\varphi}$ will denote a classical orthonormal Daubechies scaling function (of order $N$ ).

Lemma 2: For $j$ sufficiently small, the design procedures in Sections IV-A, B, C-1, and C-2 lead to filters that satisfy $Q_{j}\left(e^{i \omega}\right)=Q\left(e^{i \omega}\right) \exp \left(\epsilon_{j}\left(e^{i \omega}\right)\right)$, with $\left|\epsilon_{j}\left(e^{i \omega}\right)\right| \leq K 2^{j}$ where $K$ is a constant. In the biorthogonal cases of Sections IV-C-1 and C-2, a similar relation holds for $\tilde{Q}_{j}\left(e^{i \omega}\right)$ with respect to $\tilde{Q}\left(e^{i \omega}\right)$.

Theorem 3: The infinite product in (18) converges for every value of $\omega$. Moreover, whenever the corresponding stationary scaling function $\hat{\varphi}$ is in $L^{2}(\mathbb{R})$, the functions $\left(\hat{\varphi}_{j}\right)_{j \leq j_{0}}$ are in $L^{2}(\mathbb{R})$ and $\left\|\varphi_{j}-\varphi\right\|_{L^{2}(\mathbb{R})} \rightarrow 0$ as $j \rightarrow-\infty$.

The proofs of these results are given in the Appendix. This defines a sequence of functions $\left(\hat{\varphi}_{j}\right)_{j \leq j_{0}}$ (and $\left(\hat{\tilde{\varphi}}_{j}\right)_{j \leq j_{0}}$ in the biorthogonal case) which, by construction, satisfy the nonstationary scaling relation (3). Since there are standard procedures to determine the $L^{2}(\mathbb{R})$-membership of $\varphi[16]$, Theorem 3 tells us that the same procedures can be used to determine the $L^{2}(\mathbb{R})$-membership of the functions $\left(\varphi_{j}\right)_{j \leq j_{0}}$.

In the context of Section IV-B (respectively, Section IV-C), the compact support and the orthonormality (respectively, biorthogonality) can be shown using the same techniques as in [18], [3]. Also, hypothesis H1 (for Theorem 1) is satisfied (at sufficiently small scales, $\varphi_{j}$ and $\tilde{\varphi}_{j}$ have the same support as $\varphi$ and $\tilde{\varphi}$ respectively). In the context of Section IV-A, the interpolation property follows from the fact that, at a given scale, the autocorrelation of the orthonormal scaling function of parameter $\vec{\alpha}$ corresponds to the generalized Deslauriers-Dubuc interpolation function with $\vec{\gamma}=(\vec{\alpha}:-\vec{\alpha})$.

As a consequence of Theorem 3 , we also have the following properties. 


\section{Corollary 1:}

1) Each function $\varphi_{j}$ (respectively $\tilde{\varphi}_{j}$ ) generates a Riesz-basis. Moreover their Riesz-bounds fulfill condition $\mathbf{H 2}$.

2) $\bigcup_{j \leq j_{0}} V_{j}$ is dense in $L^{2}(\mathbb{R})$.

1) We detail the proof for the functions $\left(\varphi_{j}\right)_{j \leq j_{0}}$. The first step consists in showing that the autocorrelation sequences $a_{\varphi_{j}}$ converge uniformly toward $a_{\varphi}$ when $j \rightarrow-\infty$ :

$$
\begin{aligned}
a_{\varphi_{j}}[k]= & \left\langle\varphi_{j}, \varphi_{j}(\cdot-k)\right\rangle=\left\langle\varphi_{j}-\varphi+\varphi,\right. \\
& \left.\varphi_{j}(\cdot-k)-\varphi(\cdot-k)+\varphi(\cdot-k)\right\rangle \\
= & \left\langle\varphi_{j}-\varphi, \varphi_{j}(\cdot-k)-\varphi(\cdot-k)\right\rangle \\
& +\left\langle\varphi_{j}-\varphi, \varphi(\cdot-k)\right\rangle \\
& +\left\langle\varphi, \varphi_{j}(\cdot-k)-\varphi(\cdot-k)\right\rangle+a_{\varphi}[k] .
\end{aligned}
$$

Therefore, using the Cauchy-Schwarz inequality and the fact that $\left\|\varphi_{j}-\varphi\right\|_{L^{2}(\mathbb{R})} \rightarrow 0$ as $j \rightarrow 0$

$$
\begin{aligned}
& \left|a_{\varphi_{j}}[k]-a_{\varphi}[k]\right| \\
& \leq\left\|\varphi_{j}-\varphi\right\|_{L^{2}(\mathbb{R})}^{2}+2\left\|\varphi_{j}-\varphi\right\|_{L^{2}(\mathbb{R})}\|\varphi\|_{L^{2}(\mathbb{R})} \underset{j \rightarrow-\infty}{\longrightarrow} 0 .
\end{aligned}
$$

We point out that both autocorrelation sequences have a finite number of nonzero coefficients (and the same support for $j$ small enough). It follows that

$$
\sup _{\omega \in[-\pi, \pi]}\left|A_{\varphi_{j}}\left(e^{i \omega}\right)-A_{\varphi}\left(e^{i \omega}\right)\right| \underset{j \rightarrow-\infty}{\longrightarrow} 0 .
$$

and since $\varphi$ generates a Riesz basis, the same must be true for $\varphi_{j}$ at sufficiently small scales $j$. At higher scales, we can use an inductive argument based on the (frequencydomain) scaling relation for the autocorrelation sequences $a_{\varphi_{j}}$

$$
\begin{aligned}
A_{\varphi_{j+1}}\left(e^{i 2 \omega}\right)=\frac{1}{4}\left(\left|H_{j}\left(e^{i \omega}\right)\right|^{2} A_{\varphi_{j}}\left(e^{i \omega}\right)\right. \\
\left.+\left|H_{j}\left(-e^{i \omega}\right)\right|^{2} A_{\varphi_{j}}\left(-e^{i \omega}\right)\right) .
\end{aligned}
$$

Assuming that $\varphi_{j}$ generates a Riesz basis means that $0<$ $B_{1 j}^{2} \leq A_{\varphi_{j}}\left(e^{i \omega}\right) \leq B_{2 j}^{2}<+\infty$. In addition, $H_{j}(z)$ only has a finite number of roots, which do not comprise pairs of opposite sign. Thus there exists constants $B_{1 j+1}, B_{2 j+1}$ such that $0<B_{1 j+1}^{2} \leq A_{\varphi_{j}+1}\left(e^{i \omega}\right) \leq B_{2 j+1}^{2}<+\infty$.

2) This is a consequence of the fact that the spaces $V_{j}$ are dense in the stationary case and that $\left\|\varphi_{j}-\varphi\right\|_{L^{2}(\mathbb{R})} \rightarrow 0$ as $j \rightarrow 0$.

\section{CONCLUSION}

In this paper, we have presented a novel family of wavelet bases that generalize those introduced by Daubechies et al. They are characterized by three essential properties: they are orthonormal (respectively biorthogonal), compactly supported and the scaling functions have the ability to reproduce a predefined set of exponential polynomials.

The corresponding discrete wavelet transforms have two attractive features. First, their algorithmic implementation is straightforward: it just consists in applying Mallat's fast wavelet transform with scale-dependent filters. Second, the parameters of the exponential polynomials offer new degrees of freedom that have not been explored so far. There is good hope that these can be tuned to the specificities of certain classes of signals.

One could envisage applications in several fields, such as speech and audio processing, or neurophysiology. Indeed, these disciplines are concerned with signals that have strong harmonic components or significant exponential trends. Other examples include the raw time signals encountered in magnetic resonance imaging, RF ultrasound imaging, and optical coherence tomography. Such signals could be represented concisely using our new class of wavelet transforms.

\section{APPENDIX}

\section{A. List of the Main Notations}

Note: wherever it is applicable, the notation for the dual entities is obtained by adding a tilde " $\sim$ ". Fourier-transforms of functions are denoted with a hat "へ^" and z-transforms of discrete filters are indicated by a capital letter.

- $i$ : complex root of -1 .

- $j$ : scale parameter (finer scales as $j \rightarrow-\infty$ ).

- $\vec{\gamma}, \vec{\alpha}$ : parameters defining the exponential polynomials to be reproduced.

- $V_{j}, W_{j}$ : scaling and wavelet function spaces at scale $j$.

- $\varphi_{j}(t), \psi_{j}(t)$ : scaling and wavelet functions at scale $j$.

- $h_{j}[k], g_{j}[k]$ : scaling and wavelet filters at scale $j$.

- $x_{j}[k], y_{j}[k]$ : scaling and wavelet coefficients at scale $j$.

- $B_{1 j}, B_{2 j}$ : lower and upper Riesz bounds at scale $j$.

- $\beta_{\vec{\gamma}}(t)$ : exponential B-spline of parameter $\vec{\gamma}$.

- $r_{\vec{\gamma}}[k]$ : exponential B-spline scaling filter (up to a mutliplicative constant).

\section{B. Proof of Theorem 1}

The main idea behind the proof is to use Lemma 3 (see below) to exhibit a contradiction with hypothesis $\mathbf{H 2}$ if the desired equivalence were not true. We will use the notation $\Phi_{j}(t)=\varphi_{j}(t) e^{-2^{j} \gamma t}$.

Proof: 1) $\Rightarrow$ 2)

- As a consequence of Property 2, the functions $\left(\Phi_{j}\right)_{j \leq j_{0}}$ must be of order $L$, i.e.

$$
\forall j \leq j_{0}-1, \forall k \in \mathbb{Z} \backslash\{0\}, \hat{\Phi}_{j+1}(\omega+2 \pi k)=O\left(\omega^{L}\right) .
$$

From the scaling relation (3), we know that

$$
2 \hat{\Phi}_{j+1}(\omega)=H_{j}\left(e^{2^{j} \gamma+i \omega / 2}\right) \hat{\Phi}_{j}(\omega / 2) .
$$

Choosing $k$ of the form $2 n+1$ in (19) then yields

$$
\begin{aligned}
\forall j \leq j_{0}-1, \forall n & \in \mathbb{Z}, H_{j}\left(-e^{2^{j} \gamma+i \omega / 2}\right) \\
\times & \hat{\Phi}_{j}(\omega / 2+(2 n+1) \pi)=O\left(\omega^{L}\right) .
\end{aligned}
$$

In particular, at a given scale $j$, this means that either $H_{j}\left(-e^{2^{j} \gamma}\right)=0$, or

$$
\forall n \in \mathbb{Z}, \hat{\Phi}_{j}(2 n \pi+\pi)=0 .
$$

- Now, in contradiction to the theorem, assume that $H_{j_{1}}\left(-e^{2^{j_{1}} \gamma}\right) \neq 0$ for some $j_{1} \leq j_{0}-1$. We will show by induction on $j$ toward $-\infty$ that we can construct a sequence $\left(\omega_{j}\right)_{j \leq j_{1}}$, such that $\hat{\Phi}_{j}\left(\omega_{j}+2 n \pi\right)=0$ for all 
$n \in \mathbb{Z}$. We initialize the induction with $\omega_{j_{1}}=\pi$. Assume that property (22) holds at some scale $j+1 \leq j_{1}$. Then, at scale $j$, the scaling relation (20) yields

$\forall n \in \mathbb{Z}, H_{j}\left(e^{2^{j} \gamma+i \omega_{j+1} / 2+i n \pi}\right) \hat{\Phi}_{j}\left(\omega_{j+1} / 2+n \pi\right)=0$.

We know that either $H_{j}\left(e^{2^{j} \gamma+i \omega_{j+1} / 2}\right) \neq 0$ or $H_{j}\left(-e^{2^{j} \gamma+i \omega_{j+1} / 2}\right) \neq 0$. It follows that, if we choose $\omega_{j}$ to be either $\omega_{j+1} / 2$ or $\omega_{j+1} / 2+\pi$, then $\hat{\Phi}_{j}\left(\omega_{j}+2 n \pi\right)=0$.

- In combination with the Poisson formula, this yields (recall that $\Phi_{j}(t)$ is compactly supported)

$$
\forall j \leq j_{1}, \sum_{k \in \mathbb{Z}} e^{-i \omega_{j}(t-k)} \Phi_{j}(t-k)=0 .
$$

Dividing by $e^{-i \omega_{j} t}$ and taking the scalar product with $\Phi_{j}(t)^{*}$, we obtain $A_{\Phi_{j}}\left(e^{i \omega_{j}}\right)=0$ for all $j \leq j_{1}$. Following Lemma 3 (see below), we should then have $\left|A_{\varphi_{j}}\left(e^{i \omega_{j}}\right)\right| \rightarrow 0$ as $j \rightarrow-\infty$, but this contradicts the Riesz-basis hypothesis $\mathbf{H 2}$, stating that $\left|A_{\varphi_{j}}\left(e^{i \omega}\right)\right| \geq \mathcal{B}_{1}$ for $j \leq j_{0}$ (which holds everywhere since $a_{\varphi_{j}}$ has a finite number of nonzero coefficients).

- We have thus shown that (22) cannot hold, i.e., there exists an $n \in \mathbb{Z}$ such that $\hat{\Phi}_{j_{1}}(2 n \pi+\pi) \neq 0$. From (21), we get

$$
H_{j_{1}}\left(-e^{2^{j_{1}} \gamma+i \omega / 2}\right)=O\left(\omega^{L+1}\right)
$$

which expresses that $H_{j_{1}}(z)$ has an $L$ th-order zero at $z=$ $-e^{2^{j_{1}} \gamma}$ (remember that $H_{j_{1}}\left(-e^{2^{j_{1}} \gamma+i \omega / 2}\right)$ is infinitely differentiable since $h_{j_{1}}$ has finite support).

2) $\Rightarrow$ 1)

- Let us consider the neighborhood of $\omega=2 \pi k$ for some $j \in \mathbb{Z}$ and some $k=2^{N} p$, where $N \in \mathbb{N}$ and $p$ is an odd integer. Applying (20) $(N+1)$ times yields

$$
\begin{aligned}
& \hat{\Phi}_{j+1}(\omega+2 \pi k) \\
&= \frac{1}{2^{N+1}} \hat{\Phi}_{j-N}\left(\omega / 2^{N+1}+p \pi\right) \\
& \quad \times \prod_{n=0}^{N} H_{j-n}\left(e^{2^{j-n} \gamma+i \omega / 2^{n+1}+i 2^{N-n} p \pi}\right) .
\end{aligned}
$$

Here the last factor, $H_{j-N}\left(-e^{2^{j-N}} \gamma+i \omega / 2^{N+1}\right)$, has a zero of order $L$ at $\omega=0$. Thus $\hat{\Phi}_{j+1}(\omega+2 \pi k)=O\left(\omega^{L}\right)$. Note that this is valid for any $k=2^{N} p$ where $p$ is odd and $N \in \mathbb{N}$, i.e., for any $k \in \mathbb{Z}^{*}$.

- Now let us assume that $\hat{\Phi}_{j_{1}+1}(0)=0$ for some $j_{1} \leq j_{0}-1$. Since $H_{j}(z)$ has no pair of opposite roots, (20) implies that $\hat{\Phi}_{j}(0)=0$ for all $j \leq j_{1}$. Together with the result from the previous paragraph, the Poisson formula yields

$$
\forall j \leq j_{1}, \sum_{k \in \mathbb{Z}} \Phi_{j}(t-k)=0
$$

and therefore $A_{\Phi_{j}}(1)=0$ for all $j \leq j_{1}$. This again contradicts hypothesis $\mathbf{H} 2$ and Lemma 3.

Lemma 3: Under the assumptions of Theorem 1

$$
\sup _{\omega \in[-\pi, \pi]}\left|A_{\varphi_{j}}\left(e^{i \omega}\right)-A_{\Phi_{j}}\left(e^{i \omega}\right)\right| \underset{j \rightarrow-\infty}{\longrightarrow} 0 .
$$

$$
\text { Proof: For } j \leq j_{0} \text {, }
$$

$$
\begin{aligned}
\mid a_{\varphi_{j}} & {[k]-a_{\Phi_{j}}[k] \mid } \\
= & \mid \int_{\mathbb{R}} \varphi_{j}(t) \varphi_{j}(t-k)-\varphi_{j}(t) \\
& \times e^{-2^{j} \gamma t} \varphi_{j}(t-k) e^{-2^{j} \gamma^{*}(t-k)} \mathrm{d} t \mid \\
\leq & \int_{t_{1}}^{t_{2}}\left|\varphi_{j}(t) \varphi_{j}(t-k)\right|\left|1-e^{-2^{j} \gamma t} e^{-2^{j} \gamma^{*}(t-k)}\right| \mathrm{d} t .
\end{aligned}
$$

Thus, there exists a constant $C$ such that, for $|k|<\left\lceil t_{2}-\right.$ $\left.t_{1}\right\rceil,\left|a_{\varphi_{j}}[k]-a_{\Phi_{j}}[k]\right| \leq C\left\|\varphi_{j}\right\|_{L^{2}(\mathbb{R})} 2^{j} \leq C \mathcal{B}_{2} 2^{j}$; for all other $k$ 's, $a_{\varphi_{j}}[k]$ and $a_{\Phi_{j}}[k]$ are zero (due to hypothesis H1). It follows that:

$$
\begin{aligned}
& \left|A_{\varphi_{j}}\left(e^{i \omega}\right)-A_{\Phi_{j}}\left(e^{i \omega}\right)\right| \\
& \quad=\left|\sum_{k \in \mathbb{Z}}\left(a_{\varphi_{j}}[k]-a_{\Phi_{j}}[k]\right) e^{-i \omega}\right| \\
& \quad \leq \sum_{k \in \mathbb{Z}}\left|a_{\varphi_{j}}[k]-a_{\Phi_{j}}[k]\right| \\
& \quad \leq 2\left\lceil t_{2}-t_{1}\right\rceil C \mathcal{B}_{2} 2^{j} \underset{j \rightarrow-\infty}{\longrightarrow} 0 .
\end{aligned}
$$

\section{Proof of Property 3}

The proof uses the scaling relation for the autocorrelation sequence of exponential B-splines:

$$
\begin{aligned}
a_{\beta_{2 \vec{\alpha}}}[k] & =\left\langle\beta_{2 \vec{\alpha}}, \beta_{2 \vec{\alpha}}(\cdot-k)\right\rangle \\
& =2^{1-2 N}\left(r_{\vec{\alpha}}^{T} * r_{\vec{\alpha}} * a_{\beta_{\vec{\alpha}}}\right)[2 k]
\end{aligned}
$$

where $*$ denotes discrete convolution and $r_{\vec{\alpha}}^{T}[k]=r_{\vec{\alpha}}[-k]$. Taking the z-transform of this relation leads to (note that all sequences have finite support)

$$
\begin{aligned}
A_{\beta_{2 \vec{\alpha}}}\left(z^{2}\right)=2^{-2 N}[ & R_{\vec{\alpha}}(z) R_{\vec{\alpha}}\left(z^{-1}\right) A_{\beta_{\vec{\alpha}}}(z) \\
& \left.\quad+R_{\vec{\alpha}}(-z) R_{\vec{\alpha}}\left(-z^{-1}\right) A_{\beta_{\vec{\alpha}}}(-z)\right] .
\end{aligned}
$$

The autocorrelation sequences are real-valued and symmetric for our choice of $\vec{\alpha}$. Thus we can change the variable to $Z=$ $\left(z+z^{-1}\right) / 2$ and introduce the polynomials $A_{j}(Z) \in \mathbb{R}[Z]$, such that, for all $j \in \mathbb{Z}$

$$
A_{j}\left(\frac{z+z^{-1}}{2}\right)=A_{\beta_{2^{j} \vec{\alpha}}}(z) .
$$

Observing that $A_{\beta_{2^{j+1}}}\left(z^{2}\right)=A_{j+1}\left(2 Z^{2}-1\right)$, (23) then yields $C_{j}(Z) \frac{2^{1-2 N} A_{j}(Z)}{c_{j} A_{j+1}\left(2 Z^{2}-1\right)}+C_{j}(-Z) \frac{2^{1-2 N} A_{j}(-Z)}{c_{j} A_{j+1}\left(2 Z^{2}-1\right)}=2$.

Notice that the denominator $c_{j} A_{j+1}\left(2 Z^{2}-1\right)$ is strictly positive and bounded for $Z \in[-1,1]$, because $\beta_{2^{j+1} \vec{\alpha}}$ generates a Riesz basis (except in the ill-posed cases mentioned in Section II-D-but these are prohibited by condition Ho). The same holds true for the numerators.

We have thus found a rational fraction that satisfies a Bézoutlike relation with $C_{j}(Z)$ and is strictly positive over $[-1,1]$. 
Now the idea is to approximate it with a polynomial from the solution set given in (13). We let

$$
\mu\left(Z^{2}\right)=\frac{1}{Z C_{j}(-Z)}\left[\frac{2^{1-2 N} A_{j}(Z)}{c_{j} A_{j+1}\left(2 Z^{2}-1\right)}-D_{0 j}(Z)\right]
$$

which can be shown to be a rational fraction in the variable $Z^{2}$. Thus, from Weierstrass's theorem, we can approach it uniformly on $[-1,1]$ using polynomials of the form $\lambda\left(Z^{2}\right)$, where $\lambda(Z) \in$ $\mathbb{R}[Z]$. When the error between $\lambda$ and $\mu$ will be small enough, then $D_{0 j}(Z)+Z \lambda\left(Z^{2}\right) C_{j}(-Z)$ will also be close enough to $D_{0 j}(Z)+Z \mu\left(Z^{2}\right) C_{j}(-Z)$ (which is strictly positive), so that $D_{0 j}(Z)+Z \lambda\left(Z^{2}\right) C_{j}(-Z) \geq 0$ for $Z \in[-1,1]$.

\section{Proof of Lemma 2}

This results from two observations.

- $Q(z)$ and $\tilde{Q}(z)$ have no roots on the unit circle. This is a standard result (see, e.g., [23]).

- The filters $Q_{j}\left(e^{i \omega}\right)$ (respectively, $\tilde{Q}_{j}\left(e^{i \omega}\right)$ ) converge uniformly and exponentially fast towards $Q\left(e^{i \omega}\right)$ (respectively, $\tilde{Q}\left(e^{i \omega}\right)$ ). Indeed, at each scale $j$, we can show that the function which maps a vector $\vec{\gamma}$ to the lowest degree Bézout solution $D_{0} j(Z)$ of Section IV-A is well-defined and continuously differentiable in the neighborhood of $\vec{\gamma}=\overrightarrow{0}$. More specifically, let us denote by $\mathbf{x}_{j}$ the vector containing the coefficients of the polynomial $D_{0 j}(Z)$ at scale $j$. Then $\mathbf{x}_{j}$ is obtained as the solution of a linear system of equations of the form $\mathbf{A}_{j} \mathbf{x}_{j}=\mathbf{b}$. Similarly, the coefficients of $D_{0}(Z)$, the lowest degree Bézout solution in the stationary case, are obtained from a system of the form $\mathbf{A x}=\mathbf{b}$. Because $\left\|\mid \mathbf{A}-\mathbf{A}_{j}\right\| \|=O\left(2^{j}\right)$ and $\mathbf{A}$ is invertible, it follows that $\left\|\mathbf{x}-\mathbf{x}_{j}\right\|=O\left(2^{j}\right)$ and:

$$
\sup _{Z \in[-1,1]}\left|D_{0 j}(Z)-D_{0}(Z)\right|=O\left(2^{j}\right) .
$$

- In Section IV-A (generalized Deslauriers-Dubuc functions), we used $Q_{j}\left(e^{i \omega}\right)=e^{i \omega M / 2} D_{0 j}(\cos (\omega))$. Because $Q(z)$ has no roots on the unit circle, it follows that $Q_{j}\left(e^{i \omega}\right) / Q\left(e^{i \omega}\right)=1+O\left(2^{j}\right)$, uniformly over $\omega$.

- In Section IV-C-1 (generalized Cohen-DaubechiesFeauveau functions), $Q_{j}\left(e^{i \omega}\right)=2^{-N+1}=Q\left(e^{i \omega}\right)$ and $\tilde{Q}_{j}\left(e^{i \omega}\right)=2^{N} e^{i \omega(\tilde{N}-N) / 2} \tilde{c}_{j} D_{0}(\cos (\omega))$. Because $\tilde{Q}(z)$ has no roots on the unit circle, we get $\tilde{Q}_{j}\left(e^{i \omega}\right) / \tilde{Q}\left(e^{i \omega}\right)=1+O\left(2^{j}\right)$, uniformly over $\omega$.

- In Section IV-B (generalized orthonormal Daubechies functions), the filters $Q_{j}(z)$ are obtained from the (unique) minimum-phase factorization of $2 c_{j} D_{0 j}\left(z+z^{-1} / 2\right)$ into $Q_{j}(z) Q_{j}\left(z^{-1}\right)$. Because $Q(z)$ has no roots on the unit circle, this mapping is well-defined and continuously differentiable with respect to $D_{0 j}(Z)$, in the neighborhood of $D_{0}(Z)$. Since $D_{0 j}(Z)=D_{0}(Z)+O\left(2^{j}\right)$, we thus have $Q_{j}\left(e^{i \omega}\right) / Q\left(e^{i \omega}\right)=1+O\left(2^{j}\right)$.

- In Section IV-C-2 (generalized 9/7 functions), the filters $Q_{j}(z)$ and $\tilde{Q}_{j}(z)$ are obtained from a factorization of $2 z^{(N-\tilde{N}) / 2} \tilde{c}_{j} D_{0 j}\left(z+z^{-1} / 2\right)$ into $Q_{j}(z) \tilde{Q}_{j}\left(z^{-1}\right)$. This mapping is again well-defined and continuously differentiable in the neighborhood of $D_{0}(Z)$. From (17), moreover, $D_{0 j}(Z)$ converges exponentially fast towards $D_{0}(Z)$, which implies $Q_{j}\left(e^{i \omega}\right) / Q\left(e^{i \omega}\right)=1+O\left(2^{j}\right)$ and $\tilde{Q}_{j}\left(e^{i \omega}\right) / \tilde{Q}\left(e^{i \omega}\right)=1+O\left(2^{j}\right)$.

Because $Q_{j}\left(e^{i \omega}\right) / Q\left(e^{i \omega}\right)=1+O\left(2^{j}\right)$, we can write $Q_{j}\left(e^{i \omega}\right) / Q\left(e^{i \omega}\right)=\exp \left(\epsilon_{j}\left(e^{i \omega}\right)\right)$, where $\epsilon_{j}\left(e^{i \omega}\right)$ is such that $\epsilon_{j}\left(e^{i \omega}\right)=O\left(2^{j}\right)$ (uniformly over $\omega$ ). The same applies to $\tilde{Q}_{j}\left(e^{i \omega}\right)$ and $\tilde{Q}\left(e^{i \omega}\right)$.

\section{E. Proof of Theorem 3}

We consider the orthonormal case as an example; for the other cases, the argument stays the same but the notations must be adapted.

- Pointwise convergence: For $j$ sufficiently small, using the previous lemma, (18) can be written as three infinite products

$$
\begin{aligned}
\hat{\varphi}_{j}(\omega)= & \left(\prod_{\ell=1}^{+\infty} \frac{1}{2^{N}} R_{2^{j-\ell} \vec{\alpha}}\left(e^{i \omega / 2^{\ell}}\right)\right) \\
& \times\left(\prod_{\ell=1}^{+\infty} 2^{N-1} Q\left(e^{i \omega / 2^{\ell}}\right)\right) \\
& \times\left(\prod_{\ell=1}^{+\infty} \exp \left(\epsilon_{j-\ell}\left(e^{i \omega / 2^{\ell}}\right)\right)\right) .
\end{aligned}
$$

- The first one converges toward $\hat{\beta}_{2^{j} \vec{\alpha}}(\omega)$, the Fourier transform of an exponential B-spline of parameter $2^{j} \vec{\alpha}$.

- The second one converges to a function $\hat{q}(\omega)$, since $Q\left(e^{i \omega}\right)$ is equal to $2^{1-N}$ for $\omega=0$ and the corresponding filter has finite length (see also, [3, Lemma 3.1]).

— The infinite sum $\sum_{\ell=1}^{+\infty} \epsilon_{j-\ell}\left(e^{i \omega / 2^{\ell}}\right)$ converges absolutely because of the upper-bound $\left|\epsilon_{j}\left(e^{i \omega}\right)\right| \leq K 2^{j}$.

For larger $j$ 's, the functions $\hat{\varphi}_{j}$ are related to the previous ones by a dyadic dilation and a finite number of supplementary filter factors (see (18)).

- Convergence in $L^{2}(\mathbb{R})$ : It is known [5] that the inverse Fourier transform of $\hat{q}$ is a tempered distribution $q$. The latter is related to the stationary scaling function by

$$
\varphi(t)=(\beta * q)(t),
$$

where $\beta$ is a regular B-spline of order $N$. In our case $\varphi$ and $q$ are compactly supported, as is $\beta$. Because the $N$ th derivative of $\beta$ corresponds to an $N$ th-order finite-difference filter, we can write $q$ under the form

$$
\begin{aligned}
& q(t)=\sum_{k \in \mathbb{Z}} c[k] \varphi^{(N)}(t-k) \\
& \text { with } c[k]=\left(\begin{array}{c}
N-1+k \\
N-1
\end{array}\right) \quad \text { if } k \geq 0 \text { and } 0 \text { otherwise. }
\end{aligned}
$$

Note that locally, the sum has only a finite number of nonzero terms, since $\varphi$ is compactly supported. Hence

$$
\begin{aligned}
\left(\beta_{2^{j} \vec{\alpha}} * q\right)(t) & =\sum_{k \in \mathbb{Z}} c[k]\left(\beta_{2^{j} \vec{\alpha}} * \varphi^{(N)}\right)(t-k) \\
& =\sum_{k \in \mathbb{Z}} c[k]\left(\beta_{2^{j} \vec{\alpha}}^{(N)} * \varphi\right)(t-k) .
\end{aligned}
$$


Here, $\beta_{2^{j} \vec{\alpha}}^{(N)} * \varphi$ is in $L^{2}(\mathbb{R})$. This is best understood in the case where $\vec{\alpha}=[\alpha]$ (i.e., $N=1$ ). Indeed, in the frequency-domain, we then have

$$
\begin{aligned}
\mid \mathcal{F} & \left\{\beta_{2^{j} \vec{\alpha}}^{(N)}\right\}(\omega) \mid \\
& =\left|(i \omega) \frac{1-e^{2^{j} \alpha-i \omega}}{i \omega-2^{j} \alpha}\right| \\
& \leq\left|1-e^{2^{j} \alpha-i \omega}\right|+\left|2^{j} \alpha \beta_{2^{j} \vec{\alpha}}(\omega)\right| \\
& \leq 1+e^{2^{j} \operatorname{Re}\{\alpha\}}+2^{j}|\alpha| \frac{e^{2^{j}} \operatorname{Re}\{\alpha\}}{2^{j} \operatorname{Re}\{\alpha\}} .
\end{aligned}
$$

It follows that the Fourier transform of $\beta_{2^{j}}^{(N)}$ is bounded and the bound does not depend on $j$, for $j \leq j_{0}$ (in the general case, we just have to multiply the bounds of the corresponding first-order cases). We thus obtain $\left\|\beta_{2^{j} \vec{\alpha}}^{(N)} * \varphi\right\|_{L^{2}(\mathrm{R})} \leq C\|\varphi\|_{L^{2}(\mathrm{R})}$, where $C$ is a constant, and we know that $\varphi \in L^{2}(\mathrm{R})$. In addition, both $\beta_{2^{j} \vec{\alpha}} * q$ and $\beta_{2^{j} \vec{\alpha}}^{(N)} * \varphi$ are compactly supported. Therefore, only a finite number of terms in (24) contribute to $\beta_{2^{j}}{ }_{\alpha} * q$ over its support, from which we infer that it is a function of $L^{2}(\mathbb{R})$ as well. For $j$ small enough, we conclude that $\hat{\varphi}_{j}(\omega)=\hat{\beta}_{2^{j}} \vec{\alpha}(\omega) \hat{q}(\omega) \exp \left(\sum_{\ell=1}^{+\infty} \epsilon_{j-\ell}\left(e^{i \omega / 2^{\ell}}\right)\right)$ is in $L^{2}(\mathbb{R})$ because the last factor is bounded. Again, the functions at larger scales are related to those at smaller scales by a finite number of dilation and filtering operations; because the filters are finitely supported, the functions $\hat{\varphi}_{j}$ at larger scales are also in $L^{2}(\mathbb{R})$.

- Asymptotic behavior: The convergence toward the corresponding stationary scaling function results from the following facts.

- When the parameter vector $2^{j} \vec{\alpha} \in \mathbb{C}^{N}$ tends to zero, $\beta_{2^{j} \vec{\alpha}} * q$ converges to $\varphi$ in $L^{2}(\mathbb{R})$. Indeed, we can write that

$$
\begin{aligned}
& \left(\beta_{2^{j} \vec{\alpha}} * q\right)(t)-(\beta * q)(t) \\
& \quad=\sum_{k \in \mathbb{Z}} c[k]\left[\left(\beta_{2^{j} \vec{\alpha}}^{(N)}-\beta^{(N)}\right) * \varphi\right](t-k) .
\end{aligned}
$$

The Fourier transform of $\left(\beta_{2^{j} \vec{\alpha}}^{(N)}-\beta^{(N)}\right) * \varphi$ converges pointwise to zero. Moreover, using bounds of the type (25), one can show that its modulus is dominated by a square-integrable function, namely $|\hat{\varphi}(\omega)|$. The dominated convergence theorem thus implies that $\| \beta_{2^{j} \vec{\alpha}}^{(N)} *$ $\varphi-\beta^{(N)} * \varphi \|_{L^{2}(\mathbb{R})} \rightarrow 0$ as $j \rightarrow-\infty$. The desired convergence result follows from the compact support (and its independence from $j$ ) of the functions $\beta_{2^{j}} \vec{\alpha}^{*} q, \varphi$ and $\left(\beta_{2^{j} \vec{\alpha}}^{(N)}-\beta^{(N)}\right) * \varphi$ in (26).

— The factor $\hat{f}_{j}(\omega)=\exp \left(\sum_{\ell=1}^{+\infty} \epsilon_{j-\ell}\left(e^{i \omega / 2^{\ell}}\right)\right)$ tends to 1 uniformly as $j \rightarrow-\infty$. Thus

$$
\begin{aligned}
& \left\|\hat{\varphi}_{j}-\hat{\varphi}\right\|_{L^{2}(\mathbb{R})} \\
& =\left\|\hat{\beta}_{2^{j}} \hat{\alpha} \hat{q} \hat{f}-\hat{\beta} \hat{q}\right\|_{L^{2}(\mathbb{R})} \\
& \leq\left\|\hat{\beta}_{2^{j} \vec{\alpha}} \hat{q}\left(\hat{f}_{j}-1\right)\right\|_{L^{2}(\mathbb{R})} \\
& \quad+\left\|\hat{\beta}_{2^{j} \vec{\alpha}} \hat{q}-\hat{\beta} \hat{q}\right\|_{L^{2}(\mathbb{R})} \underset{j \rightarrow-\infty}{\longrightarrow} 0 .
\end{aligned}
$$

\section{REFERENCES}

[1] S. Mallat, A Wavelet Tour of Signal Processing. New York: Academic, 1998.

[2] Y. Meyer, Wavelets and Operators. Cambridge, U.K.: Cambridge Univ. Press, 1992.

[3] I. Daubechies, "Orthogonal bases of compactly supported wavelets," Commun. Pure Appl. Math., vol. 41, pp. 909-996, Nov. 1988.

[4] R. R. Coifman, Y. Meyer, and M. V. Wickerhauser, "Wavelet analysis and signal processing," in Wavelets and Their Applications, B. R. , Ed. New York: Jones and Bartlett, 1992, pp. 153-178.

[5] M. Unser and T. Blu, "Wavelet theory demystified," IEEE Trans. Signal Process., vol. 51, no. 2, pp. 470-483, Feb. 2003.

[6] A. Ron, "Exponential box splines," Construct. Approx., vol. 4, pp. 357-378, Dec. 1988.

[7] M. Unser and T. Blu, "Cardinal exponential splines: Part I-Theory and filtering algorithms," IEEE Trans. Signal Process., vol. 53, no. 4, pp. 1425-1438, Apr. 2005.

[8] C. de Boor, R. DeVore, and A. Ron, "On the construction of multivariate (pre)wavelets," Construct. Approx., vol. 9, pp. 123-166, Jun. 1993.

[9] I. Khalidov and M. Unser, "From differential equations to the construction of wavelet-like bases," IEEE Trans. Signal Process., vol. 54, no. 4, pp. 1256-1267, Apr. 2006.

[10] N. Dyn and D. Levin, "Analysis of asymptotically equivalent binary subdivision schemes," J. Math. Anal. Applicat., vol. 193, pp. 594-621, Jul. 1995.

[11] A. Cohen and N. Dyn, "Nonstationary subdivision schemes and multiresolution analysis," SIAM J. Math. Anal., vol. 27, no. 6, pp. 1745-1769, 1996.

[12] T. N. T. Goodman and S. L. Lee, "Convergence of nonstationary cascade algorithms," Numerische Mathematik, vol. 84, no. 1, pp. 1-33, 1999.

[13] N. Dyn, D. Levin, and A. Luzzatto, "Exponentials reproducing subdivision schemes," Found. Computat. Math., vol. 3, pp. 187-206, May 2003.

[14] G. Deslauriers and S. Dubuc, "Interpolation dyadique," in Fractals, Dimensions Non-Entières et Applications, G. Cherbit, Ed. Paris, France: Masson, 1987, pp. 44-55.

[15] W. Lawton and C. A. Micchelli, "Construction of conjugate quadrature filters with specified zeros," Numer. Algorithms, vol. 14, no. 4, pp. 383-399, 1997.

[16] G. Strang and T. Nguyen, Wavelets and Filter Banks. Wellesley, MA: Wellesley-Cambridge Press, 1996.

[17] S. Mallat, "A theory for multiresolution signal decomposition: the wavelet representation," IEEE Trans. Pattern Anal. Mach. Intell., vol. 11, pp. 674-693, Jul. 1989.

[18] A. Cohen, I. Daubechies, and J.-C. Feauveau, "Biorthogonal bases of compactly supported wavelets," Commun. Pure Appl. Math., vol. 45, no. 5, pp. 485-560, 1992.

[19] A. Antonini, M. Barlaud, P. Mathieu, and I. Daubechies, "Image coding using wavelet transforms," IEEE Trans. Image Process., vol. 1, pp. 205-220, Apr. 1992.

[20] G. Strang and G. Fix, "A Fourier analysis of the finite element variational method," in Constructive Aspects of Functional Analysis, G. Geymonat, Ed. Rome, Italy: Edizioni Cremonese, 1973, pp. 793-840.

[21] G. Deslauriers and S. Dubuc, "Symmetric iterative interpolation processes," Construct. Approx., vol. 5, no. 1, pp. 49-68, 1989.

[22] I. Daubechies, "Ten lectures on wavelets," in CBMS-NSF Regional Conf. Series in Applied Math., SIAM, 1992.

[23] J. Shen and G. Strang, "Asymptotic analysis of Daubechies polynomials," Proc. Amer. Math. Soc., vol. 124, pp. 3819-3833, Dec. 1996.

[24] M. Unser and T. Blu, "Mathematical properties of the JPEG2000 wavelet filters," IEEE Trans. Image Process., vol. 12, pp. 1080-1090, Sep. 2003.

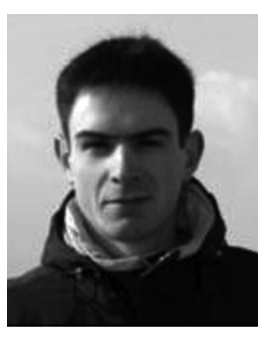

Cédric Vonesch (S'04) was born on April 14, 1981, in Obernai, France. He spent one year studying at the Ecole Nationale Supérieure des Télécommunications, Paris, France, and graduated from the EPFL, the Swiss Institute of Technology, Lausanne, in 2004.

$\mathrm{He}$ is currently with the Biomedical Imaging Group, EPFL, where he is working toward the Ph.D. degree. His research interests include multiresolution and wavelet analysis, inverse problems, and applications to bioimaging. 


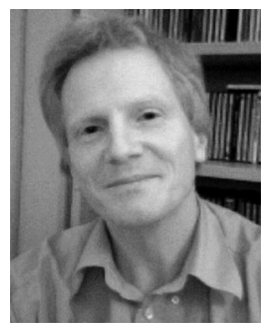

Thierry Blu (M'96-SM'06) was born in Orléans, France, in 1964. He received the "Diplôme d'ingénieur" from École Polytechnique, France, in 1986 and from the Télécom Paris (ENST), France, in 1988. In 1996, he received the Ph.D. degree in electrical engineering from ENST for a study on iterated rational filterbanks, applied to wideband audio coding.

He is with the Biomedical Imaging Group, Swiss Federal Institute of Technology (EPFL), Lausanne, on leave from France Télécom R\&D Center, Issyles-Moulineaux, France. At EPFL, he teaches the theory of Signals and Systems for Microengineering and Life Science students. His research interests include (multi)wavelets, multiresolution analysis, multirate filterbanks, interpolation, approximation and sampling theory, image denoising, psychoacoustics, optics, wave propagation, etc.

Dr. Blu was the recipient of the 2003 Best Paper Award (SP Theory and Methods) from the IEEE Signal Processing Society. Between 2002 and 2006, he was an Associate Editor for the IEEE TRANSACTIONS ON IMAGE PROCESSING and since 2006, for the IEEE TRANSACTIONS ON SIGNAL PROCESSING.

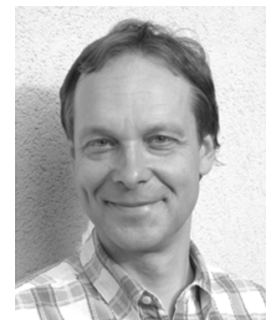

in these areas.

Dr. Unser has been actively involved with the IEEE TRANSACTIONS ON MEDICAL IMAGING, holding the positions of Associate Editor (1999-2002, 2006-present), member of the Steering Committee, and Associate Editor-in-Chief (2003-2005). He has acted as Associate Editor or member of the Editorial Board for eight more international journals, including the IEEE Signal Processing Magazine, the IEEE TRANSACTIONS ON IMAGE PROCESSING (1992-1995), and the IEEE Signal Processing LetTERS (1994-1998). He organized the first IEEE International Symposium on Biomedical Imaging (ISBI'2002). He currently chairs the Technical Committee of the IEEE-SP Society on Bio Imaging and Signal Processing (BISP), and well as the ISBI steering committee. He is the recipient of three Best Paper Awards from the IEEE Signal Processing Society.
Michael Unser (M'88-SM'94-F'99) received the M.S. (summa cum laude) and Ph.D. degrees in electrical engineering in 1981 and 1984, respectively, (EPFL), Switzerland.

From 1985 to 1997, he worked as a scientist with the National Institutes of Health, Bethesda MD. He is now professor and Director of the Biomedical Imaging Group, EPFL. His main research topics are biomedical image processing, splines, and wavelets. $\mathrm{He}$ is the author of over 120 published journal papers 